\title{
CKP Hierarchy, Bosonic Tau Function and Bosonization Formulae ${ }^{\star}$
}

\author{
Johan W. VAN DE LEUR ${ }^{\dagger}$, Alexander Yu. ORLOV ${ }^{\ddagger}$ and Takahiro SHIOTA $\S$ \\ $\dagger$ Mathematical Institute, University of Utrecht, \\ P.O. Box 80010, 3508 TA Utrecht, The Netherlands \\ E-mail: J.W.vandeLeur@uu.nl \\ $\ddagger$ Nonlinear Wave Processes Laboratory, Oceanology Institute, \\ 36 Nakhimovskii Prospect, Moscow 117851, Russia \\ E-mail: orlovs55@mail.ru \\ $\S$ Mathematics Department, Faculty of Science, Kyoto University, Kyoto 606-8502, Japan \\ E-mail: shiota@math.kyoto-u.ac.jp
}

Received January 17, 2012, in final form June 07, 2012; Published online June 22, 2012

http://dx.doi.org/10.3842/SIGMA.2012.036

\begin{abstract}
We develop the theory of CKP hierarchy introduced in the papers of Kyoto school [Date E., Jimbo M., Kashiwara M., Miwa T., J. Phys. Soc. Japan 50 (1981), 38063812] (see also [Kac V.G., van de Leur J.W., Adv. Ser. Math. Phys., Vol. 7, World Sci. Publ., Teaneck, NJ, 1989, 369-406]). We present appropriate bosonization formulae. We show that in the context of the CKP theory certain orthogonal polynomials appear. These polynomials are polynomial both in even and odd (in Grassmannian sense) variables.
\end{abstract}

Key words: integrable system; Pfaffian; Hafnian; symmetric functions; Schur type functions

2010 Mathematics Subject Classification: 17B65; 17B67; 17B69; 20G43; 81R12

\section{Introduction}

In this paper we develop the ideas of Date, Jimbo, Kashiwara and Miwa [2, 3]. In [3] it was pointed out that the tau function of the CKP hierarchy may be presented as the vacuum expectation value of bosonic fields $\phi_{n}$ which act in a bosonic Fock space, denoted by $F$ in the present paper. As it was shown in [3] the higher CKP flows are induced by the action of bosonic current algebra operators $J_{n}, n>0$. We shall show that in contrast to the familiar fermionic approach the action of the currents $J_{-n}$ on the vacuum state does not generate the whole Fock space $F$ where the original bosonic operators $\phi_{n}$ act. To generate the whole Fock space we need to add an additional fermionic field which is a sort of super-counterpart to the bosonic current. This problem is studied in Section 2. In Subsection 2.1 we introduce Fermi operators whose action on the vacuum vector complete the action of the current algebra $J_{n}$ to obtain the whole Fock space $F$. A bosonization formula which expresses the original bosonic field $\phi(z)$ in terms of the current algebra $J_{n}$ and of the fermion field $\theta(z)$ is suggested (2.11). (As a byproduct of this relation we obtain an equality (2.29) which relates Pfaffian and Hafnian expressions which earlier appeared in a quite different context [7].) Here we show that this fermionic field is a superpartner of currents and it naturally creates a dependence of CKP tau function on auxiliary odd Grassmannian parameters. Though we present a bilinear equations written in terms of super vertex operator we do not construct Lax equations with respect to odd parameters. In Section 4 we introduce new orthogonal polynomials in many variables, $\mathbf{C}_{\lambda}(\mathbf{t})$, which appear as a result of

\footnotetext{
*This paper is a contribution to the Special Issue "Geometrical Methods in Mathematical Physics". The full collection is available at http://www.emis.de/journals/SIGMA/GMMP2012.html
} 
the 'bosonization' of the basis Fock vectors of $F$, see (4.4). These polynomials depend both on CKP higher times $t_{n}$ ( $n$ odd) and the above-mentioned Grassmannian odd parameters. In certain sense these polynomials play a role similar to the role of the Schur functions in the theory of KP [16] and TL [17], and the role of the projective Schur functions in the theory of BKP [12, 19], namely, CKP tau function may be presented as a series in these polynomials over partitions (see Subsection 4.6). However in contrast to the KP and BKP cases these polynomials are not CKP tau functions themselves. At the end some combinatorial properties of $C_{\lambda}$ are discussed.

\section{CKP bosonic tau function}

In this section we follow a suggestion in [3] and describe a CKP hierarchy of PDEs starting from a collection of free bosons. This imitates their approach in the BKP case, where one starts with neutral fermions. However, this hierarchy, although related to Lie algebra $c_{\infty}$, differs from the usual CKP hierarchy, for which one takes a reduction of the KP hierarchy by assuming that the Lax operator satisfies $L^{*}=-L$; such Lax operators come from certain KP tau functions, which are fixed by some involution and where one puts the even times to zero, see e.g., [3] or [1] for more details. The hierarchy described in this paper is different and is not related to the usual CKP which comes from a reduction of KP. In the latter case one has a realization of $c_{\infty}$, for which the level is positive. Our construction realizes $c_{\infty}$ with a negative level.

\subsection{Bose-Fermi correspondence in the CKP case}

We follow a suggestion of Date, Jimbo, Kashiwara and Miwa in their paper [3] and introduce free bosons, but for convenience of notation we shift the index by $\frac{1}{2}$. So $\phi_{i}$ with $i \in \frac{1}{2}+\mathbb{Z}$ satisfy commutation relations:

$$
\phi_{i} \phi_{j}-\phi_{j} \phi_{i}=(-)^{j-\frac{1}{2}} \delta_{i,-j}
$$

The Fock space $F$, respectively $F^{*}$, is defined by

$$
\phi_{j}|0\rangle=0 \quad \text { if } j<0, \quad \text { resp. } \quad\langle 0| \phi_{j}=0 \quad \text { if } j>0,
$$

so that $F$ has as basis the vectors

$$
\left(\phi_{j_{1}}\right)^{m_{1}}\left(\phi_{j_{2}}\right)^{m_{2}} \cdots\left(\phi_{j_{n-1}}\right)^{m_{n-1}}\left(\phi_{j_{n}}\right)^{m_{n}}|0\rangle
$$

with $j_{1}>j_{2}>\cdots>j_{n-1}>j_{n}>0$ and $m_{i}$ positive integers. Defining

$$
\operatorname{deg}|0\rangle=0, \quad \operatorname{deg} \phi_{j}=j,
$$

we have a direct sum decomposition of $F$ :

$$
F=\bigoplus_{k \in \frac{1}{2} \mathbb{Z}} F_{k} \quad \text { with } \quad F_{k}=\{f \in F \mid \operatorname{deg} f=k\} .
$$

It is straightforward to check that the dimension of $F_{k}$ is given by the partition of $k$ into positive elements of $\frac{1}{2}+\mathbb{Z}$. Define the formal character as

$$
\operatorname{dim}_{q} F=\sum_{k \in \frac{1}{2} \mathbb{Z}} \operatorname{dim} F_{k} q^{k} .
$$

Then

$$
\operatorname{dim}_{q} F=\prod_{0<k \in \frac{1}{2}+\mathbb{Z}} \frac{1}{1-q^{k}} .
$$


Writing

$$
\phi(z)=\sum_{j \in \frac{1}{2}+\mathbb{Z}} \phi_{j} z^{j-\frac{1}{2}}
$$

we denote

$$
H(z):=\sum_{n \in 1+2 \mathbb{Z}} J_{n} z^{-n-1}:=-\frac{1}{2}: \phi(-z) \phi(z):
$$

where the normal ordering is defined by

$$
: \phi_{i} \phi_{j}:= \begin{cases}\phi_{i} \phi_{j} & \text { if } i \geq j \\ \phi_{j} \phi_{i} & \text { if } j>i\end{cases}
$$

In other words $J_{n}=0$ for $n$ even and

$$
J_{n}=\frac{1}{2} \sum_{j \in \frac{1}{2}+\mathbb{Z}}(-)^{j+\frac{1}{2}} \phi_{j} \phi_{-j-n} \quad \text { for } n \text { odd; }
$$

one has the following familiar commutation relations

$$
\left[J_{n}, J_{m}\right]=-\frac{n}{2} \delta_{m,-n}
$$

The elements : $\phi_{i} \phi_{j}$ : form a representation of the Lie algebra $c_{\infty}$, see e.g. [9]. However, we want to stress that its level (the value of its central element) is negative. Note also that in the commutation relations (2.7) we have the factor $-\frac{n}{2}$ instead of the usual $\frac{n}{2}$.

It is clear that

$$
J_{n}|0\rangle=\langle 0| J_{-n}=0 \quad \text { for } n>0 .
$$

By a similar argument as before, again since these are bosons, we can apply an element $J_{-n}$ infinitely many times to $|0\rangle$. Since the degree of $J_{n}$ is $-n$ we obtain that the action of this Heisenberg algebra on the vacuum vector produces in the $\operatorname{dim}_{q} F$ the partition function of partitions in only odd numbers:

$$
\prod_{0<k \in 1+2 \mathbb{Z}} \frac{1}{1-q^{k}}
$$

Now we calculate, using (2.4),

$$
\left(\prod_{0<k \in 1+2 \mathbb{Z}} \frac{1}{1-q^{k}}\right)^{-1} \operatorname{dim}_{q} F=\prod_{0<k \in \frac{1}{2}+\mathbb{Z}} \frac{1-q^{2 k}}{1-q^{k}}=\prod_{0<k \in \frac{1}{2}+\mathbb{Z}}\left(1+q^{k}\right) .
$$

This part should be explained by something else and we expect it to be fermions, at least anticommuting variables. The factor $1+q^{k}$ is related to a fermion of degree $k$. This is how we get these elements and calculate their commutation relations.

We first calculate

$$
\left[J_{n}, \phi(z)\right]=\frac{1}{2} \sum_{j, k \in \frac{1}{2}+\mathbb{Z}}(-)^{j+\frac{1}{2}}\left[\phi_{j} \phi_{-j-n}, \phi_{k}\right] z^{k-\frac{1}{2}}
$$




$$
\begin{aligned}
& =\frac{1}{2} \sum_{j, k \in \frac{1}{2}+\mathbb{Z}}(-)^{j+\frac{1}{2}}\left(\left[\phi_{j}, \phi_{k}\right] \phi_{-j-n}+\phi_{j}\left[\phi_{-j-n}, \phi_{k}\right]\right) z^{k-\frac{1}{2}} \\
& =\frac{1}{2} \sum_{j, k \in \frac{1}{2}+\mathbb{Z}}(-)^{j+\frac{1}{2}}(-)^{k-\frac{1}{2}}\left(\delta_{j,-k} \phi_{-j-n}+\delta_{j+n, k} \phi_{j}\right) z^{k-\frac{1}{2}} \\
& =\frac{1}{2} \sum_{k \in \frac{1}{2}+\mathbb{Z}} 2 \phi_{k-n} z^{k-\frac{1}{2}}=z^{n} \phi(z) .
\end{aligned}
$$

Now, using (2.7) we see that

$$
\left[J_{n}, e^{\frac{2}{m} J_{m} z^{-m}}\right]=\delta_{n,-m} z^{n} e^{\frac{2}{m} J_{m} z^{-m}} .
$$

Hence setting

$$
\theta(z):=V_{-}(z)^{-1} \phi(z) V_{+}(z)^{-1}
$$

where

$$
V_{ \pm}(z)=\exp \sum_{ \pm k>0, \text { odd }} \frac{2}{k} J_{k} z^{-k},
$$

we have, from (2.9) and (2.10),

$$
\left[J_{n}, \theta(z)\right]=0 \text {. }
$$

\subsection{Commutation relations of the $\theta(z)$ 's}

We now want to calculate the commutation relations of these $\theta(z)$ 's given in (2.11). For this we first rewrite the commutation relations (2.1) as follows:

$$
\phi(z) \phi(y)-\phi(y) \phi(z)=\delta(z-(-y)),
$$

where

$$
\delta(z-y)=z^{-1} \sum_{k \in \mathbb{Z}}\left(\frac{z}{y}\right)^{k} .
$$

Note also that

$$
\phi(-z) \phi(y)=: \phi(-z) \phi(y):-\frac{1}{z} \frac{1}{1-\frac{y}{z}} .
$$

We first show the following identities

$$
\begin{aligned}
& V_{+}(-z)^{-1} V_{-}(y)^{-1}=\frac{1-\frac{y}{z}}{1+\frac{y}{z}} V_{-}(y)^{-1} V_{+}(-z)^{-1}, \\
& \phi(-z) V_{-}(y)^{-1}=\frac{1+\frac{y}{z}}{1-\frac{y}{z}} V_{-}(y)^{-1} \phi(-z), \\
& V_{+}(-z)^{-1} \phi(y)=\frac{1+\frac{y}{z}}{1-\frac{y}{z}} \phi(y) V_{+}(-z)^{-1} .
\end{aligned}
$$

Introduce

$$
V(t)=\exp \sum_{k>0, \text { odd }} t_{k} J_{k} .
$$


Then

$$
V_{+}(z)=V\left(\frac{2}{1} z^{-1}, \frac{2}{3} z^{-3}, \frac{2}{5} z^{-5}, \ldots\right) .
$$

The first equation of (2.14) is obtained in the following way. First using (2.7) one has

$$
\begin{aligned}
V(t) V_{-}(y) & =\exp \left[\sum_{k>0, \text { odd }} t_{k} J_{k},-\sum_{\ell>0, \text { odd }} \frac{2}{\ell} y^{\ell} J_{-\ell}\right] V_{-}(y) V(t) \\
& =\exp \left(\sum_{k>0, \text { odd }} t_{k} y^{k}\right) V_{-}(y) V(t) .
\end{aligned}
$$

Combining this with (2.15) one obtains

$$
\begin{aligned}
V_{+}(-z)^{-1} V_{-}(y)^{-1} & =\exp \left(-\sum_{k>0, \text { odd }} \frac{2}{k}\left(\frac{y}{z}\right)^{k}\right) V_{-}(y)^{-1} V_{+}(-z)^{-1} \\
& =\frac{1-\frac{y}{z}}{1+\frac{y}{z}} V_{-}(y)^{-1} V_{+}(-z)^{-1}
\end{aligned}
$$

Using $\phi(-z) J_{n}=\left(J_{n}-(-z)^{n}\right) \phi(-z)$, see (2.9), we obtain the second relation in (2.14) as follows:

$$
\begin{aligned}
\phi(-z) V_{-}(y)^{-1} & =\phi(-z) \exp \left(\sum_{k>0, \text { odd }} \frac{2}{k} J_{-k} y^{k}\right)=\exp \left(\sum_{k>0, \text { odd }} \frac{2}{k}\left(J_{-k}+z^{-k}\right) y^{k}\right) \phi(-z) \\
& =V_{-}(y)^{-1} \exp \left(\sum_{k>0, \text { odd }} \frac{2}{k}\left(\frac{y}{z}\right)^{k}\right) \phi(-z)=\frac{1+\frac{y}{z}}{1-\frac{y}{z}} V_{-}(y)^{-1} \phi(-z) .
\end{aligned}
$$

The third formula is proved in a similar way.

We will also use the following identities which can be found in V. Kac's book [8]:

$$
\begin{aligned}
& (z-y) \partial_{y} \delta(z-y)=\delta(z-y), \\
& (z-y)^{k+1} \partial_{y}^{k} \delta(z-y)=0, \\
& \delta(z-y) a(z)=\delta(z-y) a(y) \\
& \partial_{y} \delta(z-y) a(z)=\partial_{y} \delta(z-y)\left(a(y)+(z-y) \partial_{y} a(y)\right) .
\end{aligned}
$$

We now calculate

$$
\begin{aligned}
\theta(-z) \theta(y) & =V_{-}(-z)^{-1} \phi(-z) V_{+}(-z)^{-1} V_{-}(y)^{-1} \phi(y) V_{+}(y)^{-1} \\
& =\frac{1-\frac{y}{z}}{1+\frac{y}{z}} V_{-}(-z)^{-1} \phi(-z) V_{-}(y)^{-1} V_{+}(-z)^{-1} \phi(y) V_{+}(y)^{-1} \\
& =V_{-}(-z)^{-1} V_{-}(y)^{-1} \phi(-z) V_{+}(-z)^{-1} \phi(y) V_{+}(y)^{-1} \\
& =\frac{1+\frac{y}{z}}{1-\frac{y}{z}} V_{-}(-z)^{-1} V_{-}(y)^{-1} \phi(-z) \phi(y) V_{+}(-z)^{-1} V_{+}(y)^{-1} \\
& =\frac{1+\frac{y}{z}}{1-\frac{y}{z}} V_{-}(-z)^{-1} V_{-}(y)^{-1}\left(: \phi(-z) \phi(y):-\frac{1}{z} \frac{1}{1-\frac{y}{z}}\right) V_{+}(-z)^{-1} V_{+}(y)^{-1} .
\end{aligned}
$$


Now replacing $z$ and $y$ by $-y$ and $-z$ respectively, gives

$$
\theta(y) \theta(-z)=\frac{1+\frac{z}{y}}{1-\frac{z}{y}} V_{-}(-z)^{-1} V_{-}(y)^{-1}\left(: \phi(-z) \phi(y):+\frac{1}{y} \frac{1}{1-\frac{z}{y}}\right) V_{+}(-z)^{-1} V_{+}(y)^{-1}
$$

and thus

$$
\begin{aligned}
\theta(-z) & \theta(y)+\theta(y) \theta(-z)=2 z \delta(z-y) V_{-}(-z)^{-1} V_{-}(y)^{-1}: \phi(-z) \phi(y): V_{+}(-z)^{-1} V_{+}(y)^{-1} \\
& +\left(\frac{1}{y} \frac{1+\frac{z}{y}}{\left(1-\frac{z}{y}\right)^{2}}-\frac{1}{z} \frac{1+\frac{y}{z}}{\left(1-\frac{y}{z}\right)^{2}}\right) V_{-}(-z)^{-1} V_{-}(y)^{-1} V_{+}(-z)^{-1} V_{+}(y)^{-1} \\
= & 4 y H(y) \delta(z-y)-\partial_{y} \delta(z-y)(y+z) V_{-}(-z)^{-1} V_{-}(y)^{-1} V_{+}(-z)^{-1} V_{+}(y)^{-1} \\
= & 4 y H(y) \delta(z-y)-2 y \partial_{y} \delta(z-y)-(z-y) \partial_{y} \delta(z-y) \\
& \times\left(1+2 y \partial_{y}\left(V_{-}(-y)^{-1}\right) V_{-}(y)^{-1}+2 y \partial_{y}\left(V_{+}(-y)^{-1}\right) V_{+}(y)^{-1}\right) \\
= & 4 y H(y) \delta(z-y)-2 y \partial_{y} \delta(z-y)-\delta(z-y)(1+4 y H(y)) \\
= & -2 y \partial_{y} \delta(z-y)-\delta(z-y)=-D_{y} \delta(z-y),
\end{aligned}
$$

where $H(y)$ is as in (2.5) (not the one in (2.28)), and $D_{y}=y \partial_{y}+\partial_{y} y$ is the Euler operator.

Now write

$$
\theta(z)=2 \sum_{i \in \frac{1}{2}+\mathbb{Z}} J_{i} z^{-i-\frac{1}{2}}
$$

Note that there is no conflict with the $J$ 's defined in (2.5), since here the $J$ 's have indices in $\frac{1}{2}+\mathbb{Z}$. It is clear that the above commutation relation (2.16) in modes gives

$$
J_{j} J_{k}+J_{k} J_{j}=(-)^{j-\frac{1}{2}} \frac{j}{2} \delta_{j,-k}, \quad j, k \in \frac{1}{2}+\mathbb{Z}
$$

(compare with (2.7)). From (2.13) we also have

$$
\left[J_{n}, J_{m}\right]=0, \quad n \in 1+2 \mathbb{Z}, \quad m \in \frac{1}{2}+\mathbb{Z} .
$$

Thus we can combine the (anti)commutation relations of all $J$ 's as follows:

$$
\left[J_{i}, J_{j}\right]_{s}=\frac{j}{2}(-1)^{\left[j-\frac{1}{2}\right]} \delta_{i,-j}
$$

where the notation $[,]_{s}$ serves for the supercommutator while $[i]$ denotes the integer part of a real number $i$. As we see, $\operatorname{deg} J_{i}=-i$ and that

$$
J_{k}|0\rangle=\left\langle 0\left|J_{-k}=0, \quad J_{-k}\right| 0\right\rangle \neq 0 \neq\langle 0| J_{k} \quad \text { for } k>0 .
$$

\subsection{Even and odd times}

Since the elements $J_{-k}$ with $k \in \frac{1}{2}+\mathbb{Z}$ anticommute among themselves, they can only appear once in

$$
J_{-k_{n}} J_{-k_{n-1}} \cdots J_{-k_{3}} J_{-k_{2}} J_{-k_{1}}|0\rangle .
$$

Such a $J_{-k}$ explains the factor $1+q^{k}$ in the $q$-dimension formula (2.8). One can identify the $J_{n}$ 's, for $n<0$ with even and odd times, i.e., with commuting variables $t_{j}, 0<j \in 1+2 \mathbb{Z}$, and Grassmann variables $t_{\frac{j}{2}}, 0<j \in 1+2 \mathbb{Z}$, and identify Fock space $F$ with the space

$$
\mathbb{C}\left[t_{2 j-1}, t_{\frac{2 j-1}{2}} ; j=1,2, \ldots\right]
$$


(or some completion of it, since we take exponentials), where one has

$$
t_{i} t_{j}-(-)^{4 i j} t_{j} t_{i}=0
$$

in particular, $t_{j}^{2}=0$ for $j \in \frac{1}{2}+\mathbb{Z}$. We will write $\mathbf{t}=\left(t_{1}, t_{\frac{1}{2}} ; t_{3}, t_{\frac{3}{2}} ; t_{5}, t_{\frac{5}{2}} ; \ldots\right)$ and use $t=$ $\left(t_{1}, t_{3}, t_{5}, \ldots\right)$ and $t_{\text {odd }}=\left(t_{\frac{1}{2}}, t_{\frac{3}{2}} ;, t_{\frac{5}{2}}, \ldots\right)$.

Let $\sigma$ be this isomorphism, sending $F$ to $\mathbb{C}\left[t_{2 j-1}, t_{\frac{2 j-1}{2}} ; j=1,2, \ldots\right]$. Then

$$
\sigma J_{-j} \sigma^{-1}=(-)^{\left[\frac{1}{2}-j\right]} \frac{j}{2} t_{j} \quad \text { and } \quad \sigma J_{j} \sigma^{-1}=\frac{\partial}{\partial t_{j}}, \quad j>0,
$$

give the field exactly in commuting and anticommuting variables $t_{k}$.

Now using the free boson-(boson+fermion) correspondence, i.e., using the vertex operator expressions for the fields

$$
\begin{aligned}
\sigma \phi(z) \sigma^{-1}= & \exp \left(\sum_{\substack{0<k \in \mathbb{Z} \\
k: \text { odd }}} t_{k} z^{k}\right) \exp \left(\sum_{\substack{0<k \in \mathbb{Z} \\
k: \text { odd }}} \frac{2}{k} \frac{\partial}{\partial t_{k}} z^{-k}\right) \\
& \times \sum_{0<j \in \mathbb{Z}}\left((2 j-1) t_{\frac{2 j-1}{2}}(-z)^{j-1}+2 \frac{\partial}{\partial t_{\frac{2 j-1}{2}}} z^{-j}\right)
\end{aligned}
$$

(where we used (2.12), (2.11), (2.17) and (2.19)), in the following subsections we shall express the bilinear identity as a hierarchy of differential equations. A similar expression for (2.20) was also found in [9].

\subsection{The CKP bilinear equation}

Following [3] we define the operator

$$
S=\sum_{k \in \frac{1}{2}+\mathbb{Z}}(-)^{k+\frac{1}{2}} \phi_{k} \otimes \phi_{-k} \equiv \oint \phi(z) \otimes \phi(-z) \frac{d z}{2 \pi i},
$$

that $S$ commutes with the action of : $\phi_{i} \phi_{j}$ : on the tensor product $F \otimes F$ of the Fock space $F$ and

$$
S(|0\rangle \otimes|0\rangle)=\sum_{k \in \frac{1}{2}+\mathbb{Z}}(-)^{k+\frac{1}{2}} \phi_{k}|0\rangle \otimes \phi_{-k}|0\rangle=0 .
$$

The CKP Hirota equation is [3]:

$$
\oint \phi(z) g|0\rangle \otimes \phi(-z) g|0\rangle d z=0,
$$

where $g$ is for instance given by (see [3]):

$$
g=\exp \left(-\sum_{m, n} c_{n m}: \phi_{n+\frac{1}{2}} \phi_{m+\frac{1}{2}}:\right) .
$$

We rewrite (2.21) as

$$
\operatorname{Res}_{z} \phi(z) g|0\rangle \otimes \phi(-z) g|0\rangle=0 .
$$

We could now use the isomorphism $\sigma$ to define this hierarchy in terms of the times t. However we will not do that yet, but concentrate first in the next subsection on the form of $\sigma(g|0\rangle)$. 


\subsection{The CKP tau function}

Now let $\mathrm{ODP}_{\mathrm{ev}}$ be the set of all partitions in an even number of odd parts, where a part may appear at most once. We call them "Odd Partitions of even length with Distinct parts" ( $\left.\mathrm{ODP}_{\mathrm{ev}}\right)$; later on we also need "Odd Partitions of odd length with Distinct parts" (ODP odd), their union $\mathrm{ODP}=\mathrm{ODP}_{\mathrm{ev}} \cup \mathrm{ODP}_{\mathrm{odd}}$, and the partition 0 . Hence for $0 \neq \alpha \in \mathrm{ODP}_{\mathrm{ev}}$ one has

$$
\alpha=\left(\alpha_{1}, \alpha_{2}, \ldots, \alpha_{2 k}\right)
$$

where all $\alpha_{i} \in 1+2 \mathbb{Z}_{\geq 0}$, and we assume

$$
\alpha_{1}>\alpha_{2}>\cdots>\alpha_{2 k} \text {. }
$$

Introduce for such partition $0 \neq \alpha \in \mathrm{ODP}_{\mathrm{ev}}$

$$
\xi_{\alpha}=t_{\frac{\alpha_{1}}{2}} t_{\frac{\alpha_{2}}{2}} \cdots t_{\frac{\alpha_{2 k}}{2}}, \quad \text { and } \quad \xi_{0}=1 .
$$

Then we can rewrite $g|0\rangle$ as

$$
\sigma(g|0\rangle)=\tau(\mathbf{t})=\sum_{\alpha \in \mathrm{ODP}_{\mathrm{ev}}} \tau_{\alpha}(t) \xi_{\alpha}
$$

Note that $\alpha \in \mathrm{ODP}_{\mathrm{ev}}$ otherwise $\langle 0|g| 0\rangle=0$. We also rewrite (2.23) as

$$
\operatorname{Res}_{z} \sum_{\alpha, \beta \in \mathrm{ODP}_{\mathrm{ev}}} \sigma \phi(z) \sigma^{-1} \tau_{\alpha}(t) \xi_{\alpha} \otimes \sigma \phi(-z) \sigma^{-1} \tau_{\beta}(t) \xi_{\beta}=0 .
$$

It is clear that we can also write

$$
\sigma\left(\phi\left(z_{0}\right) \phi\left(z_{1}\right) \cdots \phi\left(z_{k}\right)|0\rangle\right)=f(\mathbf{t})=\sum_{\alpha \in \mathrm{ODP}, \ell(\alpha) \leq k+1} f_{\alpha}(t) \xi_{\alpha} .
$$

Now let

$$
\alpha=\left(\alpha_{1}, \alpha_{2}, \ldots, \alpha_{n}\right), \quad \alpha_{i}>\alpha_{i+1} .
$$

Then clearly

$$
f_{\alpha}(t)=\left.\frac{\partial}{\partial t_{\frac{\alpha_{n}}{2}}} \frac{\partial}{\partial t_{\frac{\alpha_{n-1}}{2}}} \cdots \frac{\partial}{\partial t_{\frac{\alpha_{1}}{2}}} \sigma\left(\phi\left(z_{0}\right) \phi\left(z_{1}\right) \cdots \phi\left(z_{k}\right)|0\rangle\right)\right|_{t_{\text {odd }}=0} .
$$

Now substitute the vertex operator expression for the fields $\sigma \phi\left(z_{j}\right) \sigma^{-1}$. We thus obtain (assuming $\left|z_{i}\right|>\left|z_{j}\right|$ if $\left.i<j\right)$ :

$$
\sigma\left(\phi\left(z_{0}\right) \phi\left(z_{1}\right) \cdots \phi\left(z_{k}\right)|0\rangle\right)=\left(\prod_{0 \leq i<j \leq k} \frac{z_{i}+z_{j}}{z_{i}-z_{j}}\right) e^{\sum_{m=0}^{k} \varphi\left(t, z_{m}\right)} \sigma\left(\theta\left(z_{0}\right) \cdots \theta\left(z_{k}\right)|0\rangle\right),
$$

where

$$
\varphi(t, z)=\sum_{0<k \in \mathbb{Z}, \text { odd }} t_{k} z^{k}
$$

Then setting $\Pi(z):=\prod_{-1 \leq i<j \leq k} \frac{z_{i}+z_{j}}{z_{i}-z_{j}}$ we have

$$
f_{\alpha}(t)=\left.\Pi(z) e^{\sum_{m=0}^{k} \varphi\left(t, z_{m}\right)} \frac{\partial}{\partial t_{\frac{\alpha_{n}}{2}}} \frac{\partial}{\partial t_{\frac{\alpha_{n-1}}{2}}} \cdots \frac{\partial}{\partial t_{\frac{\alpha_{1}}{2}}} \sigma\left(\theta\left(z_{0}\right) \cdots \theta\left(z_{k}\right)|0\rangle\right)\right|_{t_{\text {odd }}=0}
$$




$$
\begin{aligned}
& =\left.\Pi(z) e^{\sum_{m=0}^{k} \varphi\left(t, z_{m}\right)} \sigma\left(J_{\frac{\alpha_{n}}{2}} J_{\frac{\alpha_{n-1}}{2}} \cdots J_{\frac{\alpha_{1}}{2}} \theta\left(z_{0}\right) \cdots \theta\left(z_{k}\right)|0\rangle\right)\right|_{t_{\mathrm{odd}}=0} \\
& =\Pi(z) e^{\sum_{m=0}^{k} \varphi\left(t, z_{m}\right)}\langle 0| J_{\frac{\alpha_{n}}{2}} J_{\frac{\alpha_{n-1}}{2} \cdots J_{\frac{\alpha_{1}}{2}} \theta\left(z_{0}\right) \cdots \theta\left(z_{k}\right)|0\rangle} \\
& =\frac{\Pi(z)}{2^{n}} e^{\sum_{m=0}^{k} \varphi\left(t, z_{m}\right)} \operatorname{Res}_{z_{-n}} \cdots \operatorname{Res}_{z_{-1}} z_{-n}^{\frac{\alpha_{n}}{2}-\frac{1}{2}} \cdots z_{-1}^{\frac{\alpha_{1}}{2}-\frac{1}{2}}\left\langle 0\left|\theta\left(z_{-n}\right) \theta\left(z_{-n+1}\right) \cdots \theta\left(z_{k}\right)\right| 0\right\rangle \\
& =\frac{\Pi(z)}{2^{n}} e^{\sum_{m=0}^{k} \varphi\left(t, z_{m}\right)} \operatorname{Res}_{z_{-n}} \cdots \operatorname{Res}_{z_{-1}} z_{-n}^{\frac{\alpha_{n}}{2}-\frac{1}{2}} \cdots z_{-1}^{\frac{\alpha_{1}}{2}-\frac{1}{2}} \operatorname{Pf}\left(\left(\frac{z_{i}-z_{j}}{\left(z_{i}+z_{j}\right)^{2}}\right)_{-n \leq i, j \leq k}\right),
\end{aligned}
$$

where Pf stands for the Pfaffian. The last equality follows from Wick's theorem and from

$$
\langle 0|\theta(y) \theta(z)| 0\rangle=\frac{y-z}{(y+z)^{2}} .
$$

Define

$$
\Gamma(\mathbf{t}):=e^{J(\mathbf{t})}, \quad J(\mathbf{t})=\sum_{0<i \in 1+2 \mathbb{Z}} t_{i} J_{i}+\sum_{0<i \in \frac{1}{2}+\mathbb{Z}} t_{i} J_{i}
$$

where $\operatorname{deg} t_{i}=i, \operatorname{deg} J(\mathbf{t})=0$. We write

$$
\Gamma(\mathbf{t})=e^{H(t)} e^{\chi\left(t_{\text {odd }}\right)}, \quad \text { where } \quad H(t):=\sum_{0<i \in 1+2 \mathbb{Z}} t_{i} J_{i}, \quad \chi\left(t_{\text {odd }}\right):=\sum_{0<i \in \frac{1}{2}+\mathbb{Z}} t_{i} J_{i} .
$$

We will now show that $f(\mathbf{t})$ is equal to

$$
\left\langle 0\left|\Gamma(\mathbf{t}) \phi\left(z_{0}\right) \phi\left(z_{1}\right) \cdots \phi\left(z_{k}\right)\right| 0\right\rangle .
$$

Using $e^{H(t)} \phi(z) e^{-H(t)}=e^{\varphi(t, z)}$ and $e^{\chi\left(t_{\text {odd }}\right)} \phi(z) e^{-\chi\left(t_{\text {odd }}\right)}=\phi(z)+\Xi(z)$, where

$$
\Xi(z)=V_{-}(z) \xi(z) V_{+}(z), \quad \xi(z)=\sum_{0<k \in \frac{1}{2}+\mathbb{Z}} k t_{k}(-z)^{k-\frac{1}{2}}
$$

we see that

$$
\begin{aligned}
\frac{\partial}{\partial t_{\frac{\alpha_{n}}{2}}} & \left.\frac{\partial}{\partial t_{\frac{\alpha_{n-1}}{2}}} \cdots \frac{\partial}{\partial t_{\frac{\alpha_{1}}{2}}}\left\langle 0\left|e^{H(t)} e^{\chi\left(t_{\text {odd }}\right)} \phi\left(z_{0}\right) \phi\left(z_{1}\right) \cdots \phi\left(z_{k}\right)\right| 0\right\rangle\right|_{t_{\mathrm{odd}}=0} \\
= & \left.\left\langle 0\left|e^{H(t)} J_{\frac{\alpha_{n}}{2}} J_{\frac{\alpha_{n-1}}{2}} \cdots J_{\frac{\alpha_{1}}{2}} e^{\chi\left(t_{\mathrm{odd}}\right)} \phi\left(z_{0}\right) \phi\left(z_{1}\right) \cdots \phi\left(z_{k}\right)\right| 0\right\rangle\right|_{t_{\mathrm{odd}}=0} \\
= & \left.\left\langle 0\left|J_{\frac{\alpha_{n}}{2}} J_{\frac{\alpha_{n-1}}{2}} \cdots J_{\frac{\alpha_{1}}{2}} e^{H(t)} e^{\chi\left(t_{\mathrm{odd}}\right)} \phi\left(z_{0}\right) \phi\left(z_{1}\right) \cdots \phi\left(z_{k}\right)\right| 0\right\rangle\right|_{t_{\mathrm{odd}}=0} \\
= & e^{\sum_{m=0}^{k} \varphi\left(t, z_{m}\right)}\langle 0| J_{\frac{\alpha_{n}}{2}} J_{\frac{\alpha_{n-1}}{2}} \cdots J_{\frac{\alpha_{1}}{2}}\left(\phi\left(z_{0}\right)+\Xi\left(z_{0}\right)\right) \\
& \times\left.\left(\phi\left(z_{1}\right)+\Xi\left(z_{1}\right)\right) \cdots\left(\phi\left(z_{k}\right)+\Xi\left(z_{k}\right)\right)|0\rangle\right|_{t_{\mathrm{odd}}=0} \\
= & e^{\sum_{m=0}^{k} \varphi\left(t, z_{m}\right)}\left\langle 0\left|J_{\frac{\alpha_{n}}{2}} J_{\frac{\alpha_{n-1}}{2}} \cdots J_{\frac{\alpha_{1}}{2}} \phi\left(z_{0}\right) \phi\left(z_{1}\right) \cdots \phi\left(z_{k}\right)\right| 0\right\rangle \\
= & e^{\sum_{m=0}^{k} \varphi\left(t, z_{m}\right)}\langle 0| J_{\frac{\alpha_{n}}{2}} J_{\frac{\alpha_{n-1}}{2}} \cdots J_{\frac{\alpha_{1}}{2}} \theta\left(z_{0}\right) \theta\left(z_{1}\right) \cdots \theta\left(z_{k}\right) \\
& \times V_{-}\left(z_{0}\right) V_{+}\left(z_{0}\right) V_{-}\left(z_{1}\right) V_{+}\left(z_{1}\right) \cdots V_{-}\left(z_{k}\right) V_{+}\left(z_{k}\right)|0\rangle
\end{aligned}
$$




$$
=\left(\prod_{0 \leq i<j \leq k} \frac{z_{i}+z_{j}}{z_{i}-z_{j}}\right) e^{\sum_{m=0}^{k} \varphi\left(t, z_{m}\right)}\left\langle 0\left|J_{\frac{\alpha_{n}}{2}} J_{\frac{\alpha_{n-1}}{2}} \cdots J_{\frac{\alpha_{1}}{2}} \theta\left(z_{0}\right) \theta\left(z_{1}\right) \cdots \theta\left(z_{k}\right)\right| 0\right\rangle=f_{\alpha}(t) .
$$

Note that from this for $k$ odd, due to Wick's rule for bosons we also have

$$
\begin{aligned}
f_{0}(t) & =e^{\sum_{m=0}^{k} \varphi\left(t, z_{m}\right)}\left\langle 0\left|\phi\left(z_{0}\right) \phi\left(z_{1}\right) \cdots \phi\left(z_{k}\right)\right| 0\right\rangle=e^{\sum_{m=0}^{k} \varphi\left(t, z_{m}\right)} \operatorname{Hf}\left(\left(\left\langle\phi\left(z_{i}\right) \phi\left(z_{j}\right)\right\rangle\right)_{0 \leq i, j \leq k}\right) \\
& =e^{\sum_{m=0}^{k} \varphi\left(t, z_{m}\right)} \operatorname{Hf}\left(\left(\frac{1}{z_{i}+z_{j}}\right)_{0 \leq i, j \leq k}\right),
\end{aligned}
$$

where Hf stands for the Hafnian. The Hafnian of a symmetric matrix $A$ of even order is defined as follows

$$
\operatorname{Hf}(A):=\sum_{\sigma} A_{\sigma(1), \sigma(2)} A_{\sigma(3), \sigma(4)} \cdots A_{\sigma(2 k-1), \sigma(2 k)},
$$

where the sum runs over all permutations $\sigma$ of $\{1, \ldots, 2 k\}$ satisfying

$$
\sigma(2 i-1)<\sigma(2 i), \quad \sigma(1)<\sigma(3)<\cdots<\sigma(2 k-1) .
$$

As one can see the Hafnian contains $1 \cdot 3 \cdot 5 \cdots(2 k-1)=:(2 k-1)$ !! terms.

Remark 1. Comparing this with $f_{0}$ in (2.26), we have a new proof for the identity

$$
\operatorname{Pf}\left(\left(\frac{z_{i}-z_{j}}{\left(z_{i}+z_{j}\right)^{2}}\right)_{1 \leq i, j \leq 2 k}\right)=\prod_{1 \leq i<j \leq 2 k} \frac{z_{i}-z_{j}}{z_{i}+z_{j}} \operatorname{Hf}\left(\left(\frac{1}{z_{i}+z_{j}}\right)_{1 \leq i, j \leq 2 k}\right)
$$

of $[7]$.

Since $g|0\rangle$, where $g$ is given by (2.22), is a possibly infinite linear combination of

$$
\phi_{j_{1}} \phi_{j_{2}} \cdots \phi_{j_{2 k}}|0\rangle
$$

which can be obtained by taking residues of the expression in (2.24), one deduces that

$$
\sigma(g|0\rangle)=\tau(\mathbf{t})=\left\langle 0\left|e^{H(t)} e^{\chi\left(t_{\text {odd }}\right)} g\right| 0\right\rangle=\sum_{\alpha} \tau_{\alpha}(t) \xi_{\alpha}
$$

and

$$
\tau_{\alpha}(t)=\left\langle\alpha\left|e^{H(t)} g\right| 0\right\rangle,
$$

where

$$
\langle\alpha|=\langle 0| J_{\frac{\alpha_{n}}{2}} J_{\frac{\alpha_{n-1}}{2}} \cdots J_{\frac{\alpha_{1}}{2}} .
$$

\subsection{A CKP wave function}

Now we want to study $\phi(z) g|0\rangle$. Consider the expression

$$
\begin{aligned}
\sigma(\phi(z) g|0\rangle) & =\sigma \phi(z) \sigma^{-1} \sigma(g|0\rangle)=\sigma \phi(z) \sigma^{-1} \tau(\mathbf{t}) \\
& =e^{\varphi(t, z)} \sum_{0<j \in \mathbb{Z}}\left((2 j-1) t_{\frac{2 j-1}{2}}(-z)^{j-1}+2 \frac{\partial}{\partial t_{\frac{2 j-1}{2}}} z^{-j}\right) \times
\end{aligned}
$$




$$
\times \exp \left(\sum_{0<k \in \mathbb{Z}, \text { odd }} \frac{2}{k} \frac{\partial}{\partial t_{k}} z^{-k}\right) \tau(\mathbf{t}),
$$

where (2.20) was used.

Clearly, one also has

$$
\sigma(\phi(z) g|0\rangle)=\left\langle 0\left|e^{H(t)} e^{\chi\left(t_{\text {odd }}\right)} \phi(z) g\right| 0\right\rangle .
$$

We now write $\sigma(\phi(z) g|0\rangle)$ as

$$
\sigma(\phi(z) g|0\rangle)=\sum_{\alpha \in \mathrm{ODP}_{\text {odd }}} g_{\alpha}(t, z) \xi_{\alpha}
$$

Now substitute this in (2.23), omitting the tensor symbol and writing $s_{j}$ for $t_{j}$ in the right-hand side of the tensor product, we obtain, that for every $\alpha, \beta \in \mathrm{ODP}_{\text {odd }}$ the coefficient of $\xi_{\alpha} \eta_{\beta}$ is equal to

$$
\operatorname{Res}_{z} g_{\alpha}(t, z) g_{\beta}(s,-z)=0 \text {. }
$$

Now we want to express $g_{\alpha}(t, z)$ in terms of the $\tau_{\beta}(t)$ 's.

It will be convenient to introduce some more notation here. Let

$$
\alpha=\left(\alpha_{1}, \alpha_{2}, \ldots, \alpha_{k}\right) \in \mathrm{ODP}
$$

where all $\alpha_{i} \in 1+2 \mathbb{Z}_{\geq 0}$, and we assume

$$
\alpha_{1}>\alpha_{2}>\cdots>\alpha_{k} .
$$

Now let $\nu \in 1+2 \mathbb{Z}_{\geq 0}, \nu \notin \alpha$, i.e.,

$$
\nu \notin\left\{\alpha_{1}, \alpha_{2}, \ldots, \alpha_{k}\right\}
$$

and

$$
\alpha_{i}>\nu>\alpha_{i+1}
$$

then we define an "addition" as follows

$$
\alpha \cup \nu:=\left(\alpha_{1}, \alpha_{2}, \ldots, \alpha_{i}, \nu, \alpha_{i+1}, \ldots, \alpha_{k}\right) .
$$

Note that the notation $\alpha+\beta$ was used differently in [11] where it was defined as $\left(\alpha_{1}+\beta_{1}, \alpha_{2}+\right.$ $\left.\beta_{2}, \ldots\right)$.

In a similar way the subtraction $\alpha \backslash \alpha_{i}$ for $\alpha_{i} \in \alpha$ is defined by

$$
\alpha \backslash \alpha_{i}=\left(\alpha_{1}, \alpha_{2}, \ldots, \alpha_{i-1}, \alpha_{i+1}, \ldots, \alpha_{k}\right) .
$$

Then for

$$
\alpha=\left(\alpha_{1}, \alpha_{2}, \ldots, \alpha_{k}\right) \in \mathrm{ODP}_{\text {odd }},
$$

hence $k$ odd, we find

$$
g_{\alpha}(t, z)=e^{\varphi(t, z)} \exp \left(\sum_{0<j \in \mathbb{Z}, \text { odd }} \frac{2}{j} \frac{\partial}{\partial t_{j}} z^{-j}\right) \times
$$




$$
\times\left(\sum_{i=1}^{k}(-)^{i-1} \alpha_{i} \tau_{\alpha \backslash \alpha_{i}}(t)(-z)^{\frac{\alpha_{i}-1}{2}}+2 \sum_{\nu \in 1+2 \mathbb{Z}_{\geq 0}, \nu \notin \alpha} s(\nu, \alpha) \tau_{\alpha \cup \nu}(t) z^{-\frac{\nu+1}{2}}\right),
$$

where

$$
s(\nu, \alpha)=(-1)^{\left|\left\{\alpha_{i} \in \alpha \mid \alpha_{i}>\nu\right\}\right|} .
$$

In particular for $\alpha=(1)=1$ we find

$$
g_{1}(t, z)=e^{\varphi(t, z)} \exp \left(\sum_{0<j \in \mathbb{Z}, \text { odd }} \frac{2}{j} \frac{\partial}{\partial t_{j}} z^{-j}\right)\left(\tau_{0}(t)+2 \sum_{\nu \in 1+2 \mathbb{Z}, \nu>1} \tau_{(\nu, 1)}(t) z^{-\frac{\nu+1}{2}}\right),
$$

We now want to calculate $g_{\alpha}(t, z)$ as some expectation value. Using (2.33), we have

$$
\begin{aligned}
g_{\alpha}(t, z) & =\left.\frac{\partial}{\partial t_{\frac{\alpha_{n}}{2}}} \frac{\partial}{\partial t_{\frac{\alpha_{n-1}}{2}}} \cdots \frac{\partial}{\partial t_{\frac{\alpha_{1}}{2}}}\left\langle 0\left|e^{H(t)} e^{\chi\left(t_{\text {odd }}\right)} \phi(z) g\right| 0\right\rangle\right|_{t_{\text {odd }}=0} \\
& =\frac{1}{2^{n}}\left\langle 0\left|\theta_{\frac{\alpha_{n}}{2}} \theta_{\frac{\alpha_{n-1}}{2}} \cdots \theta_{\frac{\alpha_{1}}{2}} e^{H(t)} \phi(z) g\right| 0\right\rangle=\left\langle\alpha\left|e^{H(t)} \phi(z) g\right| 0\right\rangle .
\end{aligned}
$$

where we have used (2.32).

Now concentrating on (2.35) and divide this by $(-)^{\frac{\alpha_{1}-1}{2}} \alpha_{1} \tau_{\alpha \backslash \alpha_{1}}(t)$, this gives

$$
\begin{aligned}
& w_{\alpha}(t, z)=\hat{w}_{\alpha}(t, z) z^{\frac{\alpha_{1}-1}{2}} e^{\varphi(t, z)}, \quad \text { where } \\
& \hat{w}_{\alpha}(t, z)=(-z)^{-\frac{\alpha_{1}-1}{2}} \frac{g_{\alpha}(t, z) e^{-\varphi(t, z)}}{\alpha_{1} \tau_{\alpha \backslash \alpha_{1}}(t)}=1+O\left(z^{-1}\right),
\end{aligned}
$$

which we call the wave function corresponding to $\alpha \in$ ODP. Now using (2.31) and (2.36), one also has

$$
w_{\alpha}(t, z)=\frac{(-)^{\frac{\alpha_{1}-1}{2}}}{\alpha_{1}} \frac{\left\langle\alpha\left|e^{H(t)} \phi(z) g\right| 0\right\rangle}{\left\langle\alpha \backslash \alpha_{1}|g| 0\right\rangle} .
$$

\subsection{A bilinear identity for the wave function and a Lax equation}

Using the definition of the wave function (2.37) we can rewrite (2.34) into a bilinear identity for these wave functions:

$$
\begin{aligned}
\operatorname{Res}_{z} w_{\alpha}(t, z) w_{\beta}(s,-z) & \\
& =\operatorname{Res}_{z} \hat{w}_{\alpha}(t, z) \hat{w}_{\beta}(s,-z) e^{\varphi(t, z)-\varphi(s, z)} z^{\frac{\alpha_{1}+\beta_{1}-2}{2}}=0, \quad \alpha, \beta \in \mathrm{ODP}_{\text {odd }} .
\end{aligned}
$$

Let us take $\alpha=\beta$ and $\alpha_{1}=\beta_{1}=n, n$ odd. Then equation (2.38) takes the form

$$
\operatorname{Res}_{z} \hat{w}_{\alpha}(t, z) \hat{w}_{\alpha}(s,-z) e^{\varphi(t, z)-\varphi(s, z)} z^{n-1}=0, \quad \alpha_{1}=\beta_{1}=n, \quad n \text { odd, }
$$

which coincides with equations (5) and $(12)_{n}(n$ odd) of [3] where $w(x, k)$ should be compared with our $e^{\varphi(t, z)} w_{\alpha}(t, z)$.

Now write for $\alpha=(1)=1$

$$
w(t, z)=w_{1}(t, z), \quad \text { and } \quad \hat{w}(t, z)=\hat{w}_{1}(t, z)
$$


then we can see this as the Date, Jimbo, Kashiwara, Miwa CKP wave function as in [3], since in particular

$$
\operatorname{Res}_{z} \hat{w}(t, z) \hat{w}(s,-z) e^{\varphi(t, z)-\varphi(s, z)}=0 .
$$

We can rewrite the wave functions as follows

$$
w_{\alpha}(t, z)=\hat{w}_{\alpha}(t, \partial) \partial^{\frac{\alpha_{1}-1}{2}} e^{\varphi(t, z)},
$$

where $\partial=\frac{\partial}{\partial x}=\frac{\partial}{\partial t_{1}}$. Then $\hat{w}_{\alpha}(t, \partial)$ is a pseudo differential operator of order 0 . Note that

$$
w_{\alpha}(t,-z)=\hat{w}_{\alpha}(t, \partial) \partial^{\frac{\alpha_{1}-1}{2}} e^{-\varphi(t, z)} .
$$

Now we use the following known lemma (see, e.g., [10]):

Lemma 1. For pseudodifferential operators $P(t, \partial)$ and $Q(t, \partial)$ we have

$$
\begin{aligned}
& \operatorname{Res}_{z}\left(P(t, \partial) \cdot \exp \left(\sum_{0<j \in \mathbb{Z}, \text { odd }} t_{j} z^{j}\right)\right)\left(Q(t, \partial) \cdot \exp \left(-\sum_{0<j \in \mathbb{Z}, \text { odd }} t_{j} z^{j}\right)\right) \\
& =\operatorname{Res}_{\partial} P(t, \partial) \cdot Q(t, \partial)^{*}=0 .
\end{aligned}
$$

where the conjugation and $\operatorname{Res}_{\partial}$ are defined on monomials respectively as $\left(a(x) \partial^{k}\right)^{*}=(-\partial)^{k} a(x)$ and $\operatorname{Res}_{\partial} a(x) \partial^{k}=a(x) \delta_{k,-1}$.

Taking $P=\hat{w}$ and $Q=\partial^{n} \hat{w}, n=0,1,2, \ldots$, by (2.39) we obtain that

$$
\left(\hat{w}_{\alpha}(t, \partial) \partial^{\frac{\alpha_{1}+\beta_{1}-2}{2}} \hat{w}_{\beta}(t, \partial)^{*}\right)_{-}=0
$$

Now we take $\alpha=\beta=(1)=1$. Then, since $\hat{w}(t, \partial)=1+\sum_{k=1}^{\infty} w_{k}(t) \partial^{-k}$, one obtains that

$$
\hat{w}(t, \partial)^{*}=\hat{w}(t, \partial)^{-1} .
$$

Start again with (2.39) and differentiate this equation in $t_{k}$ for $k$ odd; then one gets

$$
\operatorname{Res}_{z}\left(\frac{\partial \hat{w}(t, z)}{\partial t_{k}}+\hat{w}(t, z) z^{k}\right) \exp \left(\sum_{0<j \in \mathbb{Z}, \text { odd }} t_{j} z^{j}\right) w(s,-z)=0 .
$$

Again using the fundamental Lemma and (2.41), we deduce

$$
\left(\left(\frac{\partial \hat{w}(t, \partial)}{\partial t_{k}}+\hat{w}(t, \partial) \partial^{k}\right) W(t, \partial)^{-1}\right)_{-}=0
$$

which gives the Sato-Wilson equation:

$$
\frac{\partial \hat{w}(t, \partial)}{\partial t_{k}}=-\left(\hat{w}(t, \partial) \partial^{k} \hat{w}(t, \partial)^{-1}\right)_{-} \hat{w}(t, \partial) .
$$

This is equivalent to the following equation for the wave function

$$
\frac{\partial w(t, z)}{\partial t_{k}}=\left(\hat{w}(t, \partial) \partial^{k} \hat{w}(t, \partial)^{-1}\right)_{+} w(t, z) .
$$

Introducing the Lax operator

$$
L(t, \partial)=\hat{w}(t, \partial) \partial \hat{w}(t, \partial)^{-1}=\partial+\sum_{k=1}^{\infty} u_{k}(t) \partial^{-k},
$$

then (2.41) induces $L^{*}=-L$ and from the Sato-Wilson equation one derives the following CKP Lax equation:

$$
\frac{\partial L(t, \partial)}{\partial t_{k}}=\left[\left(L(t, \partial)^{k}\right)_{+}, L(t, \partial)\right]
$$




\section{Bilinear identity in super notations}

In the previous section we obtained a wave function. In this section our approach will be slightly different. We want to superize, i.e., obtain a supersymmetric wave function that also include the Grassmannian times $t_{i}$ with $i \in \frac{1}{2}+\mathbb{Z}$ and the corresponding bilinear equation for this super wave function (3.11). Let us point out that in this way we shall re-write results of the previous section using super notations. We regard this an important step, which might be very fundamental for the further development of the theory. However, unfortunately we were not able to obtain Lax equations with respect to odd Grassmannian times in this setting.

\subsection{Super vertex operator}

Recall the super commutation relations (2.18) and the super times (2.18) and the definition of $\Gamma(\mathbf{t})$ in $(2.27)$. It is convenient to introduce an auxiliary parameter $\zeta$, which is a Grassmannian variable, an odd counterpart to $z: \zeta^{2}=0, z \zeta=\zeta z, \operatorname{deg} z=2 \operatorname{deg} \zeta=-1$.

Introduce the following "superfermionic" fields

$$
\begin{aligned}
& \Phi(z, \zeta):=2 \sum_{n \in \mathbb{Z}} \zeta \frac{z^{-2 n-1}}{2 n+1} J_{2 n+1}-2 \sum_{0 \neq n \in \mathbb{Z}} \frac{z^{-n}}{n} J_{n+\frac{1}{2}}+2 J_{\frac{1}{2}} \log z, \\
& \Theta(z, \zeta):=\left(\frac{\partial}{\partial \zeta}+\zeta \frac{\partial}{\partial z}\right) \Phi(z, \zeta) .
\end{aligned}
$$

Here $\frac{\partial}{\partial \zeta}+\zeta \frac{\partial}{\partial z}$ is a superderivative which will be denoted by $D_{z, \zeta}, D_{z, \zeta}^{2}=\frac{\partial}{\partial z}$. As we see $\operatorname{deg} \Phi=-\frac{1}{2}$ while $\operatorname{deg} \Theta=0$.

Let $: e^{\Theta(z, \zeta)}$ : denote $e^{\Theta_{-}(z, \zeta)} e^{\Theta_{+}(z, \zeta)}$ where $\Theta_{ \pm}$denotes the splitting of $\Theta$ in series in respectively positive/negative powers of $z^{-1}$. One can verify

$$
: e^{\Theta\left(z_{1}, \zeta_{1}\right)}:: e^{\Theta\left(z_{2}, \zeta_{2}\right)}:=e^{\Theta-\left(z_{1}, \zeta_{1}\right)+\Theta_{-}\left(z_{2}, \zeta_{2}\right)} e^{\Theta_{+}\left(z_{1}, \zeta_{1}\right)+\Theta_{+}\left(z_{2}, \zeta_{2}\right)}\left(\frac{z_{1}+z_{2}}{z_{1}-z_{2}}+\frac{\zeta_{1} \zeta_{2}}{z_{1}+z_{2}}\right) .
$$

It follows from $\zeta^{2}=0$ that

$$
: e^{\Theta(z, \zeta)}:=V_{-}(z) V_{+}(z)+\zeta \phi(z)
$$

Then

$$
\begin{aligned}
& \phi(z)=\frac{\partial}{\partial \zeta}: e^{\Theta(z, \zeta)}:
\end{aligned}
$$

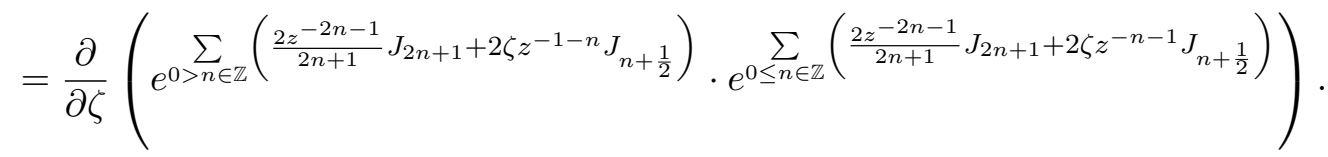

Using the isomorphism $\sigma$, see (2.19), we can express

$$
\sigma \Theta_{-}(z, \zeta) \sigma^{-1}=\sum_{0<i \in 1+2 \mathbb{Z}} z^{i} t_{i}+\zeta \sum_{0<i \in \frac{1}{2}+\mathbb{Z}} i(-z)^{i-\frac{1}{2}} t_{i}=: \varphi(\mathbf{t}, z, \zeta),
$$

and

$$
\sigma \Theta_{+}(z, \zeta) \sigma^{-1}=2 \sum_{0<n \in \mathbb{Z}} \frac{z^{1-2 n}}{2 n-1} \frac{\partial}{\partial t_{2 n-1}}+2 \zeta \sum_{0<n \in \mathbb{Z}} z^{-n} \frac{\partial}{\partial t_{n-\frac{1}{2}}} .
$$


Now

$$
\sigma \phi(z) \sigma^{-1}=\frac{\partial}{\partial \zeta} \Upsilon(\mathbf{t}, z, \zeta),
$$

where

$$
\begin{aligned}
& \Upsilon(\mathbf{t}, z, \zeta):=e^{\varphi(\mathbf{t}, z, \zeta)} \Upsilon_{+}(z, \zeta) \\
& \Upsilon_{+}(z, \zeta)=\exp \left(2 \sum_{0<i \in 1+2 \mathbb{Z}} \frac{z^{-i}}{i} \frac{\partial}{\partial t_{i}}+2 \zeta \sum_{0<i \in \frac{1}{2}+\mathbb{Z}} z^{-i-\frac{1}{2}} \frac{\partial}{\partial t_{i}}\right)
\end{aligned}
$$

\subsection{Tau function and super wave function}

Recall (2.30), (2.31) the tau function as a function of a collection of both even and odd time variables as follows

$$
\tau(\mathbf{t}):=\langle 0|\Gamma(\mathbf{t}) g| 0\rangle=\sum_{\alpha} \tau_{\alpha}(t) \xi_{\alpha} .
$$

As we can see

$$
\tau(\mathbf{t})=\tau^{\mathrm{CKP}}(t)+\cdots=\tau_{0}(t)+\cdots,
$$

where the dots mean terms vanishing when we substitute $t_{\frac{1}{2}}=t_{\frac{3}{2}}=\cdots=0$. We put

$$
W(\mathbf{t}, z, \zeta):=\frac{1}{\tau(\mathbf{t})}\left\langle 0\left|\Gamma(\mathbf{t}): e^{\Theta(z, \zeta)}: g\right| 0\right\rangle
$$

to be the wave function.

From (3.4) we can write

$$
W(\mathbf{t}, z, \zeta):=\frac{\Upsilon(\mathbf{t}, z, \zeta) \tau(\mathbf{t})}{\tau(\mathbf{t})}=e^{\varphi(\mathbf{t}, z, \zeta)}\left(1+O\left(\frac{1}{z}\right)\right)
$$

where $\varphi(\mathbf{t}, z, \zeta)$ was defined in (3.3). Let us mark that if $t_{\text {odd }}=0$ then $\varphi(\mathbf{t}, z, \zeta)$ coincides with $\varphi(t, z, \zeta)$ defined in $(2.25)$.

Note that since $\phi(z)=\frac{\partial}{\partial \zeta}: e^{\Theta(z, \zeta)}$ : it follows from (2.35) and (2.36) that for $\alpha=\left(\alpha_{1}, \ldots, \alpha_{n}\right)$

$$
g_{\alpha}(t, z)=\left.\left(\frac{\partial}{\partial \zeta} \frac{\partial}{\partial t_{\frac{\alpha_{n}}{2}}} \cdots \frac{\partial}{\partial t_{\frac{\alpha_{1}}{2}}} \Upsilon(\mathbf{t}, \zeta, z) \tau(\mathbf{t})\right)\right|_{t_{\mathrm{odd}}=0},
$$

where we remind that in our notations: $t=\left(t_{1}, t_{3}, t_{5}, \ldots\right)$ and $\mathbf{t}=\left(t_{1}, t_{\frac{1}{2}} ; t_{3}, t_{\frac{3}{2}} ; t_{5}, t_{\frac{5}{2}} ; \ldots\right)$.

\subsection{Super Miwa variables}

Denote $\mathbf{z}=\left(z_{1}, \zeta_{1} ; \ldots ; z_{k}, \zeta_{k}\right)$, where $\zeta_{i}$ are Grassmannian odd and $z_{i}$ are Grassmannian even variables, $\zeta_{i}^{2}=0$, and $\operatorname{deg} z_{i}=-1, \operatorname{deg} \zeta_{i}=-\frac{1}{2}$. Let $[\mathbf{z}]:=\left(t_{1}, t_{\frac{1}{2}} ; t_{3}, t_{\frac{3}{2}} ; t_{5}, t_{\frac{5}{2}} ; \ldots\right)$, where

$$
t_{2 n+1}=\frac{2}{(2 n+1)} \sum_{i=1}^{k} \frac{1}{z_{i}^{2 n+1}}, \quad t_{n+\frac{1}{2}}=2 \sum_{i=1}^{k} \frac{\zeta_{i}}{z_{i}^{n}} .
$$


For $k=1$ we shall write $[\mathbf{z}]$ as $[z, \zeta]$. Then

$$
W(\mathbf{t}, z, \zeta):=e^{\varphi(\mathbf{t}, z, \zeta)} \frac{\tau(\mathbf{t}-[z, \zeta])}{\tau(\mathbf{t})} .
$$

In general

$$
\langle 0| \Gamma(\mathbf{t}-[\mathbf{z}])=\mathrm{D}(\mathbf{z})^{-1}\langle 0| \prod_{i=1}^{k}: e^{\Theta\left(z_{i}, \zeta_{i}\right)}: \Gamma(\mathbf{t}),
$$

where

$$
\mathrm{D}(\mathbf{z})=\mathrm{D}_{k}(\mathbf{z}):=\left\langle 0\left|: e^{\Theta\left(z_{1}, \zeta_{1}\right)}: \cdots: e^{\Theta\left(z_{k}, \zeta_{k}\right)}:\right| 0\right\rangle,
$$

which is antisymmetric function with respect to the permutation of pairs $\left(z_{i}, \zeta_{i}\right)$. For instance

$$
\mathrm{D}_{2}(\mathbf{z})=\frac{z_{1}+z_{2}}{z_{1}-z_{2}}+\frac{\zeta_{1} \zeta_{2}}{z_{1}+z_{2}} .
$$

We have

$$
\mathrm{D}_{k}(\mathbf{z})^{-1}=\left(\prod_{a<b} \frac{z_{a}-z_{b}}{z_{a}+z_{b}}\right) \sum_{n=0}^{[k / 2]}(-1)^{n} \sum_{\substack{\alpha \in \mathrm{ODP}_{\mathrm{ev}} \\ \ell(\alpha)=2 n}} \zeta_{\alpha} \operatorname{Pf}\left[\frac{z_{\alpha_{i}}-z_{\alpha_{j}}}{\left(z_{\alpha_{i}}+z_{\alpha_{j}}\right)^{2}}\right]
$$

\subsection{Bilinear identity for the super wave function $W$}

The bilinear equation (2.34),

$$
\operatorname{Res}_{z} g_{\alpha}\left(t^{\prime}, z\right) g_{\beta}(t,-z)=0,
$$

may be written in superized form

$$
\operatorname{Ber}_{z, \zeta}\left(\left(\frac{\partial}{\partial \zeta} W\left(\mathbf{t}^{\prime}, z, \zeta\right)\right) W(\mathbf{t},-z, \mp \zeta)\right)=0,
$$

where $\operatorname{Ber}_{z, \zeta} f(z, \zeta):=\operatorname{Res}_{z} \frac{\partial f(z, \zeta)}{\partial \zeta}$. The validity of (3.11) follows directly from (2.23), (3.7), (3.2) and the definition of the Berezinian Ber.

\subsection{Bilinear identity as identities for super PDOs}

Let us re-write super-bilinear identities (3.11) in form of identities for (super version of) pseudodifferential operators (PDO). We will do it in a way similar to the KP case where PDO operators naturally appear in the framework of KP bilinear identity [4].

Now notice that (3.8) yields

$$
W(\mathbf{t}, z, \zeta)=: \hat{W}(\mathbf{t}, z, \zeta) e^{\varphi(\mathbf{t}, z, \zeta)}=:\left(\hat{W}^{(0)}(\mathbf{t}, z)+\zeta \hat{W}^{(1)}(\mathbf{t}, z)\right) e^{\varphi(\mathbf{t}, z, \zeta)}
$$

where (3.5) provides

$$
\hat{W}^{(1)}(\mathbf{t}, z)=\sum_{n>0} \hat{W}_{n}^{(1)}(\mathbf{t}) z^{-n}=O\left(\frac{1}{z}\right), \quad \hat{W}^{(0)}(\mathbf{t}, z)=\sum_{n \geq 0} \hat{W}_{n}^{(0)}(\mathbf{t}) z^{-n}=1+O\left(\frac{1}{z}\right) .
$$


For a simplification we shall denote $x=t_{1}$ and $\xi=\frac{1}{2} t_{\frac{1}{2}}$, thus $\varphi(\mathbf{t}, z, \zeta)=z x+\zeta \xi+\cdots$, and we have

$$
\partial_{x} e^{\varphi(\mathbf{t}, z, \zeta)}=z e^{\varphi(\mathbf{t}, z, \zeta)}, \quad \partial_{\xi} e^{\varphi(\mathbf{t}, z, \zeta)}=-\zeta e^{\varphi(\mathbf{t}, z, \zeta)} .
$$

Let $D:=D_{x, \xi}=\partial_{\xi}+\xi \partial_{x}, D^{2}=\partial_{x}$. Then $\partial_{\xi}=D-\xi D^{2}$. Below we consider the action of odd negative powers of $D$ on the exponentials $e^{z x+\zeta \xi}$, provided we define $\partial^{n} e^{z x}:=z^{n} e^{z x}, n \in \mathbb{Z}$. To do this we write $D^{1-2 n}=D \partial^{-n}$. In such a way we write

$$
D^{2 n} \cdot e^{\varphi(\mathbf{t}, z, \zeta)}=z^{n} e^{\varphi(\mathbf{t}, z, \zeta)}, \quad D^{2 n+1} \cdot e^{\varphi(\mathbf{t}, z, \zeta)}=z^{n}\left(-\zeta+\xi D^{2}\right) e^{\varphi(\mathbf{t}, z, \zeta)}, \quad n \in \mathbb{Z} ;
$$

in particular

$$
\zeta e^{\varphi(\mathbf{t}, z, \zeta)}=-\left(D-\xi D^{2}\right) \cdot e^{\varphi(\mathbf{t}, z, \zeta)} .
$$

Let us introduce

$$
\begin{aligned}
K(\mathbf{t}, D) & :=\sum_{n \geq 0} K_{n}(\mathbf{t}) D^{-n}:=\hat{W}^{(0)}(\mathbf{t}, \partial)-\hat{W}^{(1)}(\mathbf{t}, \partial) \frac{\partial}{\partial \xi} \\
& =\hat{W}^{(0)}\left(\mathbf{t}, D^{2}\right)-\hat{W}^{(1)}\left(\mathbf{t}, D^{2}\right)\left(D-\xi D^{2}\right) \\
& =1+\hat{W}_{1}^{(1)}(\mathbf{t}) \xi+\sum_{n \geq 1}\left(\hat{W}_{n}^{(0)}(\mathbf{t})+\hat{W}_{n+1}^{(1)}(\mathbf{t}) \xi\right) D^{-2 n}-\sum_{n \geq 1} \hat{W}_{n}^{(1)}(\mathbf{t}) D^{1-2 n},
\end{aligned}
$$

where negative powers of $D$ are to be understood in the sense of (3.13).

From (3.14), (3.15) and (3.12) it follows that

$$
W(\mathbf{t}, z, \zeta)=K(\mathbf{t}, D) \cdot e^{\varphi(\mathbf{t}, z, \zeta)} .
$$

In the Lemma below star means the conjugation in the algebra of super PDOs with properties $(a b)^{*}= \pm b^{*} a^{*}$, where - is taken iff both $a$ and $b$ are odd. We have $\left(\partial_{x}\right)^{*}=-\partial_{x},\left(\partial_{\xi}\right)^{*}=-\partial_{\xi}$. For instance $D^{*}=-\partial_{\xi}+\left(-\partial_{x}\right) \xi=-D,\left(D^{2}\right)^{*}=-D^{*} D^{*}=-\partial,(\xi \zeta)^{*}=-\zeta \xi=\xi \zeta$, and $\left(a D^{n}\right)^{*}=(-1)^{[n / 2]} D^{n} a$ if $a$ is odd.

We define $\operatorname{Res}_{D} \sum_{n \in \mathbb{Z}} f_{n}(x, \xi) D^{n}:=f_{-1}(x, \xi)$. Notice that

$$
\operatorname{Res}_{D} f(x, \xi) D^{-1} g(x, \xi)= \pm \operatorname{Res}_{D} f(x, \xi) g(x, \xi) D^{-1}
$$

where + and - are taken if $g$ is respectively even and odd.

Lemma 2. Let $P(D)=\sum_{n} P_{n}(x, \xi) D^{n}$ be an odd and $Q(D)=\sum_{n} Q_{n}(x, \xi) D^{n}$ an even PDO. Then

$$
\begin{aligned}
& \operatorname{Ber}_{z, \zeta}\left(P(D) \cdot e^{z x+\zeta \xi}\right)\left(Q(D) \cdot e^{-z x-\zeta \xi}\right)=\operatorname{Res}_{D} P(D)(Q(D))^{*} \\
& \operatorname{Ber}_{z, \zeta}\left(P(D) \cdot e^{z x+\zeta \xi}\right)\left(Q(D) \cdot e^{-z x+\zeta \xi}\right)=\operatorname{Res}_{D} P(D)(Q(-D))^{*} .
\end{aligned}
$$

Proof. Let

$$
P(D)=\sum_{n \in \mathbb{Z}}\left(P_{n}^{(0)} D^{2 n}+P_{n}^{(1)} D^{2 n} D^{-1}\right), \quad Q(D)=\sum_{n \in \mathbb{Z}}\left(Q_{n}^{(0)} D^{2 n}+Q_{n}^{(1)} D^{2 n} D^{-1}\right),
$$

where $P_{n}^{(0)}, Q_{n}^{(1)}$ are odd and $P_{n}^{(1)}, Q_{n}^{(0)}$ are even. Then

$$
(Q(D))^{*}=\sum_{n \in \mathbb{Z}}\left((-1)^{n} D^{2 n} Q_{n}^{(0)}-(-1)^{n} D^{-1} D^{2 n} Q_{n}^{(1)}\right)
$$

where we used $\left(D^{*}\right)^{2 n}=(-1)^{n} D^{2 n},\left(D^{-1}\right)^{*}=\left(D^{-2} D\right)^{*}=(D)^{-1}$. 
Using (3.16), we find that the right-hand side of (3.17) is equal to

$$
\sum_{n \in \mathbb{Z}}(-1)^{n}\left(P_{n}^{(1)} Q_{-n}^{(0)}+P_{n}^{(0)} Q_{-n}^{(1)}\right) .
$$

Next consider the left-hand side of (3.17). We have

$$
\begin{aligned}
& P(D) \cdot e^{z x+\zeta \xi}=\sum_{n \in \mathbb{Z}}\left(P_{n}^{(0)} z^{n}+P_{n}^{(1)} z^{n-1}(\zeta+z \xi)\right) e^{z x+\zeta \xi}, \\
& Q(D) \cdot e^{-z x-\zeta \xi}=\sum_{n \in \mathbb{Z}}\left(Q_{n}^{(0)}(-z)^{n}-Q_{n}^{(1)}(-z)^{n-1}(\zeta+z \xi)\right) e^{-z x-\zeta \xi} .
\end{aligned}
$$

The evaluation of the Ber of the product of these two results in (3.19).

A similar calculation yields (3.18).

We re-write (3.11) as

$$
\operatorname{Ber}_{z, \zeta}\left(K\left(\mathbf{t}^{\prime}, D\right) \cdot \frac{\partial}{\partial \zeta} e^{\varphi\left(\mathbf{t}^{\prime}, z, \zeta\right)}\right)\left(K(\mathbf{t}, D) \cdot e^{\varphi(\mathbf{t},-z, \mp \zeta)}\right)=0 .
$$

Taking into account

$$
\frac{\partial}{\partial \zeta} e^{\varphi(\mathbf{t}, z, \zeta)}=-\sum_{n \geq 0}\left((-1)^{n}\left(n+\frac{1}{2}\right) t_{n+\frac{1}{2}} \partial^{n}\right) \cdot e^{\varphi(\mathbf{t}, z, \zeta)},
$$

where $\partial:=\frac{\partial}{\partial t_{1}}=D^{2}$, and $\varphi(\mathbf{t}, z, \zeta)$ is as in $(3.3)$, we obtain

$$
\begin{aligned}
& \operatorname{Res}_{D}\left(K\left(\mathbf{t}^{\prime}, D\right) \cdot\left(\sum_{n \geq 0}(-1)^{n}\left(n+\frac{1}{2}\right) t_{n+\frac{1}{2}}^{\prime} D^{2 n}\right)\right. \\
& \left.\times e^{ \pm\left(D-\xi D^{2}\right) \sum_{n \geq 0}\left(n+\frac{1}{2}\right) t_{n+\frac{1}{2}} D^{2 n}} \cdot K^{*}(\mathbf{t}, \pm D)\right)=0,
\end{aligned}
$$

which results in

$$
\left(K(\mathbf{t}, D) \cdot\left(\sum_{n \geq 0}(-1)^{n}\left(n+\frac{1}{2}\right) t_{n+\frac{1}{2}} D^{2 n}\right) e^{ \pm\left(D-\xi D^{2}\right) \sum_{n \geq 0}\left(n+\frac{1}{2}\right) t_{n+\frac{1}{2}} D^{2 n}} \cdot K^{*}(\mathbf{t}, \pm D)\right)_{-}=0,
$$

where the subscript - means the taking of projection on series with negative powers. Thus

$$
L_{ \pm}:=K(\mathbf{t}, D) \cdot\left(\sum_{n \geq 0}(-1)^{n}\left(n+\frac{1}{2}\right) t_{n+\frac{1}{2}} D^{2 n}\right) e^{ \pm\left(D-\xi D^{2}\right) \sum_{n \geq 0}\left(n+\frac{1}{2}\right) t_{n+\frac{1}{2}} D^{2 n}} \cdot K^{*}(\mathbf{t}, \pm D)
$$

are differential operators. These equations are basically equivalent to the set of equations (2.40).

\section{Related symmetric functions}

In this section we want to introduce polynomial functions, $C_{\lambda}$, related to the basis vectors $|\lambda\rangle$ of the bosonic Fock space $F$ as the image of the mapping $\sigma$ described in the Subsection 2.3. These are polynomials in Grassmannian even and odd variables t. In super Miwa variables $z_{i}$, $\zeta_{i}, i=1, \ldots, k$ these functions are symmetric with respect to the action of the permutation group $S_{k}$ on the set of pairs $\left(z_{i}, \zeta_{i}\right)$, and polynomial with respect to variables $\zeta_{i}$ and $x_{i}:=z_{i}^{-1}$.

These polynomials may be considered as analogues of the celebrated Schur (and projective Schur) functions which are related to Fock space of charged (resp. neutral) fermions. The theoretic field construction of new functions allows to derive certain properties which are similar to the properties of the Schur and the projective Schur functions. 


\subsection{Polynomials $\mathrm{C}_{\lambda}$}

Let us introduce suitable notations for the basis of bosonic Fock vectors in $F$ (see (2.3)) and in $F^{*}$ labeled by partitions whose parts are odd numbers

$$
|\lambda\rangle:=\frac{1}{d_{\lambda}} \phi_{\frac{\lambda_{1}}{2}} \cdots \phi_{\frac{\lambda_{k}}{2}}|0\rangle, \quad\langle\lambda|:=\frac{1}{d_{\lambda}}\langle 0| \phi_{-\frac{\lambda_{k}}{2}} \cdots \phi_{-\frac{\lambda_{1}}{2}},
$$

where $\lambda=\left(\lambda_{1}, \lambda_{2}, \ldots, \lambda_{k}\right)$ is a set of odd numbers and $\lambda_{1} \geq \lambda_{2} \geq \cdots \geq \lambda_{k}>0, \ell(\lambda):=k=$ $1,2, \ldots$ The set of partitions with odd parts will be denoted by OP.

Note that the above vectors $\langle\lambda|$ differ from the vector $\langle\alpha|$ as defined in (2.32), that is the reason why we write $\lambda$ here to avoid this confusion.

In this section we shall use, besides the parts $\lambda_{i}$ of partitions $\lambda$, also the variables $n_{i}=$ $0,1,2, \ldots$ related to odd numbers $\lambda_{i}$ as follows

$$
\lambda_{i}=: 2 n_{i}+1 .
$$

The sum $|\lambda|:=\lambda_{1}+\cdots+\lambda_{k}$ is called the weight of the partition $\lambda$. Here the factor $d_{\lambda}$ for $\lambda \in \mathrm{OP}$ is defined by

$$
\left(d_{\lambda}\right)^{2}:=(-1)^{\sum_{i=1}^{k} n_{i}} \prod_{i=1,2,3, \ldots} m_{2 i-1} !=\prod_{i=1,2,3, \ldots} m_{2 i-1} !(-1)^{m_{4 i-1}}=(-1)^{\frac{1}{2}(|\lambda|-\ell(\lambda))} \prod_{i=1,3,5, \ldots} m_{i} !,
$$

where $m_{i}=m_{i}(\lambda)$ is the number of parts of $\lambda$ equal to $i$ (or, the same, the multiplicity of $i$ ). (Then we can denote the partition by its frequency notation $\lambda=\left(1^{m_{1}} 3^{m_{3}} 5^{m_{5}} \cdots\right)$ ). For instance $d_{\left(1^{n} 3^{m} 5^{k}\right)}=n ! m !(-1)^{m} k !$.

From (2.1),

$$
\phi_{-\frac{1}{2} \lambda_{i}} \phi_{\frac{1}{2} \lambda_{j}}-\phi_{\frac{1}{2} \lambda_{j}} \phi_{-\frac{1}{2} \lambda_{i}}=(-)^{n_{j}} \delta_{\lambda_{i}, \lambda_{j}}
$$

we see that, for partitions $\lambda$ and $\mu$, we have the ortho-normality condition

$$
\langle\lambda \mid \mu\rangle=\delta_{\mu, \lambda}, \quad \lambda, \mu \in \mathrm{OP} .
$$

Let us introduce the following functions

$$
\mathrm{C}_{\lambda}(\mathbf{t}):=\sigma(|\lambda\rangle):=\langle 0|\Gamma(\mathbf{t})| \lambda\rangle, \quad \lambda \in \mathrm{OP} .
$$

These functions are weighted polynomials of weight $\frac{1}{2}|\lambda|$ in the variables $\mathbf{t}:=\left(t_{1}, t_{\frac{1}{2}} ; t_{3}, t_{\frac{3}{2}} ; \ldots\right)$ where $\operatorname{deg} t_{j}:=j$. For example

$$
\mathrm{C}_{(1)}=t_{\frac{1}{2}}, \quad \mathrm{C}_{\left(1^{2}\right)}=\frac{t_{1}}{\sqrt{2}}
$$

We evaluate $C_{\lambda}$ in case all (Grassmannian) odd variables vanish: $t_{n+\frac{1}{2}}=0, n=0,1,2, \ldots$ (Recall that in this case we denote $\mathbf{t}$ as $t=\left(t_{1}, t_{3}, \ldots\right)$ ).

Remark 2. Weighted polynomial functions are often presented as symmetric functions of some variables $x_{1}, \ldots, x_{N}$, where the number of variables may be irrelevant. In our case we put

$$
t_{n}=\frac{1}{n} \sum_{i=1}^{N}\left(x_{i}^{n}-\left(-x_{i}\right)^{n}\right), \quad n=1,2,3, \ldots,
$$


where all even-labeled $t_{n}$ vanish. For $n$ odd we write

$$
t_{n}=\frac{2}{n} \sum_{i=1}^{N} x_{i}^{n}, \quad n=1,3, \ldots,
$$

where $x_{i}=\frac{1}{z_{i}}, i=1, \ldots, N$. Below by polynomial functions in Miwa variables we mean polynomial functions in the variables $x_{i}=\frac{1}{z_{i}}$.

Then $C_{\lambda}(t)$ vanishes if $\ell(\lambda)$ is $\operatorname{odd}^{1}$. If $\ell(\lambda)$ is even, then by Wick's theorem

$$
\mathrm{C}_{\lambda}(t)=\operatorname{Hf}\left[\mathrm{C}_{\left(\lambda_{i}, \lambda_{j}\right)}(t)\right], \quad \lambda \in \mathrm{OP}_{\mathrm{e}},
$$

where $\mathrm{OP}_{\mathrm{e}}$ is the set of all partitions with even number of odd parts, and as we shall see

$$
\mathrm{C}_{\left(\lambda_{i}, \lambda_{j}\right)}(t)=\frac{1}{d_{\left(\lambda_{i} \mid \lambda_{j}\right)}} s_{\left(n_{i} \mid n_{j}\right)}(t),
$$

where $s_{\lambda}$ is the Schur function, and $(n \mid m)$ is a one-hook partition in the Frobenius notations, see [11]. Indeed, first, from

$$
\left[J_{2 n-1}, \phi_{i}\right]=\phi_{i-2 n+1}
$$

(cf. (2.9)) it follows

$$
\phi_{i}(t):=\Gamma(t) \phi_{i} \Gamma(t)^{-1}=\sum_{n=0}^{\infty} h_{n}(t) \phi_{i-n},
$$

where $h_{n}$ are complete symmetric functions [11]. Now from (2.1), (2.2) we obtain

$$
\left\langle 0\left|\phi_{n_{1}+\frac{1}{2}}(t) \phi_{n_{2}+\frac{1}{2}}(t)\right| 0\right\rangle=\sum_{n=0}^{\lambda_{1}}(-1)^{n_{2}-n} h_{n}(t) h_{n_{1}+n_{2}+1-n}(t),
$$

while Schur function evaluated on a one-hook partition is (see Chapter I, § 3, Example 9 in [11])

$$
s_{\left(n_{1} \mid n_{2}\right)}(t)=h_{n_{1}+1}(t) e_{n_{2}}(t)-h_{n_{1}+2}(t) e_{n_{2}-1}(t)+\cdots+(-)^{n_{2}} h_{n_{1}+n_{2}+1}(t),
$$

where $e_{n}$ are elementary symmetric functions. Then taking into account that for $t=\left(t_{1}, t_{3}, \ldots\right)$ of form (4.5) we get the equality $h_{m}(t) \equiv e_{m}(t)$, we obtain (4.6).

Thus we get

$$
C_{\lambda}(t)=\left\{\begin{array}{lll}
\frac{1}{d_{\lambda}} \operatorname{Hf}\left[s_{\left(n_{i} \mid n_{j}\right)}(t)\right] & \text { if } \ell(\lambda) & \text { even } \\
0 & \text { if } \ell(\lambda) & \text { odd }
\end{array}\right.
$$

It follows from $(4.7)$ and from $s_{\lambda}(-t)=(-1)^{|\lambda|} s_{\lambda^{t r}}(t)$ that

$$
\mathrm{C}_{\lambda}(-t)=(-1)^{\frac{1}{2}(|\lambda|+\ell(\lambda))} \mathrm{C}_{\lambda}(t) .
$$

Next, if all Grassmannian odd variables except $t_{\frac{1}{2}}$ vanish, we obtain

$$
C_{\lambda}(t)= \begin{cases}\frac{1}{d_{\lambda}} \operatorname{Hf}\left[s_{\left(n_{i} \mid n_{j}\right)}(t)\right] & \text { if } \ell(\lambda) \text { even, } \\ t_{\frac{1}{2}} \frac{1}{d_{\lambda}} \operatorname{Hf}[\tilde{S}] & \text { if } \ell(\lambda)=2 n-1 \text { odd }\end{cases}
$$

\footnotetext{
${ }^{1}$ We need to make difference between odd numbers and odd numbers in the Grassmannian sense. In the last case we shall necessarily say Grassmannian odd number.
} 
where $\tilde{S}$ is $2 n \times 2 n$ symmetric matrix

$$
\tilde{S}_{i j}=\tilde{S}_{j i}:= \begin{cases}s_{\left(n_{i} \mid n_{j}\right)}(t) & \text { if } 1 \leq i<j \leq 2 n-1, \\ s_{\left(n_{i}\right)}(t) & \text { if } 1 \leq i<j=2 n .\end{cases}
$$

Recall that $t=\left(t_{1}, t_{3}, t_{5}, \ldots\right)$ and that $\lambda_{i}$ are related to $n_{i}$ via $(4.2)$.

In general case, where the odd Grassmannian variables do not vanish, we can see that $C_{\lambda}$ is of even Grassmannian parity in case $\ell(\lambda)$ is even, and it is of odd Grassmannian parity in case $\ell(\lambda)$ is odd. We can write

$$
\mathrm{C}_{\lambda}(t)= \begin{cases}\frac{1}{d_{\lambda}} \operatorname{Hf}\left[s_{\left(n_{i} \mid n_{j}\right)}(t)\right]+C_{e} & \text { if } \ell(\lambda) \text { even, } \\ t_{\frac{1}{2}} \frac{1}{d_{\lambda}} \operatorname{Hf}[\tilde{S}]+C_{o} & \text { if } \ell(\lambda)=2 n-1 \text { odd },\end{cases}
$$

where $C_{e}$ and $C_{o}$ are polynomials in odd Grassmannian variables of the order $\ell(\lambda), C_{e}$ starts with quadratic terms, while $C_{o}$ starts with cubic ones. This follows from the consideration of (2.24) in Subsection 2.1 .

\subsection{Orthogonality}

One can verify the equality, using (2.27)

$$
\langle 0|\Gamma(\mathbf{t})| \lambda\rangle=\langle\lambda|\bar{\Gamma}(-\mathbf{t})| 0\rangle,
$$

where

$$
\bar{\Gamma}(\mathbf{t}):=e^{\bar{J}(\mathbf{t})}, \quad \bar{J}(\mathbf{t})=\sum_{0<i \in 1+2 \mathbb{Z}} t_{i} J_{-i}+\sum_{0<i \in \frac{1}{2}+\mathbb{Z}} t_{i} J_{-i}
$$

(keep the order in the products of Grassmannian odd variables, which we label by semi-integer subscripts).

Thanks to (4.3) we can write

$$
\langle 0| \Gamma(\mathbf{t})=\langle 0|+\sum_{\lambda \in \mathrm{OP}} \mathrm{C}_{\lambda}(\mathbf{t})\langle\lambda|
$$

On the other hand due to (4.9)

$$
\bar{\Gamma}(\mathbf{t})|0\rangle=|0\rangle+\sum_{\lambda \in \mathrm{OP}}|\lambda\rangle \mathrm{C}_{\lambda}(-\mathbf{t})
$$

and to $(2.18)$ we obtain

$$
\langle 0|\Gamma(\mathbf{t}) \bar{\Gamma}(\overline{\mathbf{t}})| 0\rangle=e^{-\frac{1}{2} \sum_{n=1,3,5, \ldots} n t_{n} \bar{t}_{n}-\frac{1}{2}} \sum_{m=0,1,2, \ldots}(-1)^{m}\left(m+\frac{1}{2}\right) t_{m+\frac{1}{2}} \bar{t}_{m+\frac{1}{2}},
$$

where $\mathbf{t}$ and $\overline{\mathbf{t}}$ are two independent sets of variables:

$$
\mathbf{t}=\left(t_{1}, t_{\frac{1}{2}} ; t_{3}, t_{\frac{3}{2}} ; t_{5}, t_{\frac{5}{2}} ; \ldots\right), \quad \overline{\mathbf{t}}=\left(\bar{t}_{1}, \bar{t}_{\frac{1}{2}} ; \bar{t}_{3}, \bar{t}_{\frac{3}{2}} ; \bar{t}_{5}, \bar{t}_{\frac{5}{2}} ; \ldots\right) .
$$

On the other hand, due to $(2.27),(4.10)$ and (4.3) we obtain the following analogue of CauchyLittlewood identity:

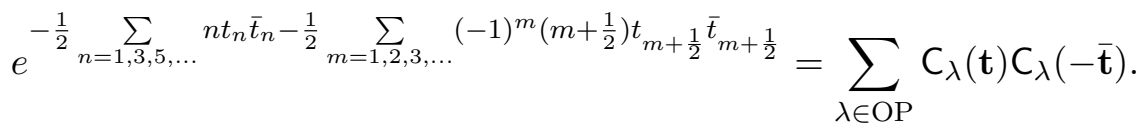


From the last equality we obtain

$$
\Gamma(\mathbf{t})=1+\sum_{\lambda \in \mathrm{OP}} \mathrm{C}_{\lambda}(\mathbf{t}) \mathrm{C}_{\lambda}(\mathbf{J}), \quad \bar{\Gamma}(\mathbf{t})=1+\sum_{\lambda \in \mathrm{OP}} \mathrm{C}_{\lambda}(\overline{\mathbf{J}}) \mathrm{C}_{\lambda}(-\overline{\mathbf{t}}),
$$

where

$$
\begin{aligned}
& \mathbf{J}:=\left(2 J_{1}, 4 J_{\frac{1}{2}} ; \frac{2}{3} J_{3},-\frac{4}{3} J_{\frac{3}{2}} ; \frac{2}{5} J_{5}, \frac{4}{5} J_{\frac{5}{2}} ; \frac{2}{7} J_{7},-\frac{4}{7} J_{\frac{7}{2}} ; \ldots\right), \\
& \overline{\mathbf{J}}:=\left(-2 J_{-1},-4 J_{-\frac{1}{2}} ;-\frac{2}{3} J_{-3}, \frac{4}{3} J_{-\frac{3}{2}} ;-\frac{2}{5} J_{-5},-\frac{4}{5} J_{-\frac{5}{2}} ;-\frac{2}{7} J_{-7}, \frac{4}{7} J_{-\frac{7}{2}} ; \ldots\right)
\end{aligned}
$$

and therefore

$$
|\lambda\rangle=\mathrm{C}_{\lambda}(\overline{\mathbf{J}})|0\rangle, \quad\langle\lambda|=\langle 0| \mathrm{C}_{\lambda}(\mathbf{J}) .
$$

Let $f(\mathbf{t})$ and $g(\mathbf{t})$ be series in the variables $\left\{t_{i}\right\}$. We introduce the following scalar product

$$
\langle f, g\rangle:=\langle 0|f(\mathbf{J}) g(\overline{\mathbf{J}})| 0\rangle .
$$

In particular due to $(2.18)$

$$
\left\langle t_{i}, t_{j}\right\rangle=\left\langle 0\left|J_{i} J_{-j}\right| 0\right\rangle=-\frac{j}{2}(-1)^{\left[-j-\frac{1}{2}\right]} \delta_{i, j},
$$

where $[a]$ denotes the integer part of $a$ (i.e., $a=[a]+\epsilon$ where $0 \leq \epsilon \leq 1$ for $a<0$ ).

It follows from (4.13) and (4.3) that the polynomials form an orthonormal basis in the scalar product (4.14):

$$
\left\langle\mathrm{C}_{\lambda}, \mathrm{C}_{\mu}\right\rangle=\left\langle 0\left|\mathrm{C}_{\lambda}(\mathbf{J}) \mathrm{C}_{\mu}(\overline{\mathbf{J}})\right| 0\right\rangle=\delta_{\lambda, \mu}
$$

\subsection{Polynomials $\mathrm{C}_{\lambda}$ in super Miwa variables}

If we want to rewrite polynomials as symmetric functions symmetric with respect to the action of symmetric group on pairs $\left(z_{i}, \zeta_{i}\right)$ of super Miwa variables $\mathbf{z}=\left(z_{1}, \zeta_{1}, \ldots, z_{k}, \zeta_{k}\right)$ (3.9), we present $C_{\lambda}$ as

$$
\mathrm{C}_{\lambda}(\mathbf{z}):=\mathrm{C}_{\lambda}(-[\mathbf{z}])=\left\langle 0\left|: e^{\Theta\left(z_{1}, \zeta_{1}\right)}: \cdots: e^{\Theta\left(z_{k}, \zeta_{k}\right)}:\right| \lambda\right\rangle \mathrm{D}(\mathbf{z})^{-1}
$$

in terms of super vertex operators (3.1). Here

$$
\mathrm{D}(\mathbf{z})=\mathrm{D}_{k}(\mathbf{z}):=\left\langle 0\left|: e^{\Theta\left(z_{1}, \zeta_{1}\right)}: \cdots: e^{\Theta\left(z_{k}, \zeta_{k}\right)}:\right| 0\right\rangle
$$

is antisymmetric function with respect to the permutation of pairs $\left(z_{i}, \zeta_{i}\right), \mathrm{D}(\mathbf{z})^{-1}$, see $(3.10)$. Let us note that if $\ell(\lambda)>k$ the polynomial $C_{\lambda}$ does not depend on the odd Grassmannian variables $\zeta_{i}$, and vanish for $\ell(\lambda)$ odd. Example. For $k=1$ we obtain

$$
\mathrm{C}_{\lambda}(z, \zeta)= \begin{cases}\frac{2}{d_{\lambda}} z^{-\frac{1}{2}|\lambda|}(2 \ell(\lambda)-1) ! ! & \text { if } \quad \ell(\lambda) \text { even, } \\ \frac{\zeta}{d_{\lambda}} z^{-\frac{1}{2}|\lambda|-\frac{1}{2}} & \text { if } \quad \ell(\lambda)=1, \\ 0 & \text { if } \quad \ell(\lambda)>1, \text { odd }\end{cases}
$$

where we use the formula $s_{(n \mid m)}(x)=2 x^{n+m+1}$.

In Miwa variables the Cauchy-Littlewood identity (4.12) is written as

$$
\sum_{\lambda \in \mathrm{OP}} \mathrm{C}_{\lambda}(\mathbf{z}) \mathrm{C}_{\lambda}(-\overline{\mathbf{z}})=\prod_{i, j} \frac{1-z_{i} \bar{z}_{j}}{1+z_{i} \bar{z}_{j}}\left(1-\zeta_{i} \bar{\zeta}_{j} \frac{1-z_{i} \bar{z}_{j}}{\left(1+z_{i} \bar{z}_{j}\right)^{2}}\right) .
$$


Proof. Using (3.9), namely

$$
t_{2 n+1}=\frac{2}{(2 n+1)} \sum_{i=1}^{k} \frac{1}{z_{i}^{2 n+1}}, \quad t_{m+\frac{1}{2}}=2 \sum_{i=1}^{k} \frac{\zeta_{i}}{z_{i}^{m}}
$$

we obtain

$$
\begin{aligned}
& e^{-\frac{1}{2} \sum_{m=1,2,3, \ldots}(-1)^{m}\left(m+\frac{1}{2}\right) t_{m+\frac{1}{2}} \bar{t}_{m+\frac{1}{2}}}=\prod_{i, j}\left(1-2 \zeta_{i} \bar{\zeta}_{j} \sum_{m=1,2,3, \ldots}(-1)^{m}\left(m+\frac{1}{2}\right) z_{i}^{-m} \bar{z}_{j}^{-m}\right) \\
& =\prod_{i, j}\left(1+2 \zeta_{i} \bar{\zeta}_{j} D_{z_{i}} \sum_{m=1,2,3, \ldots}(-1)^{m} z_{i}^{-m} \bar{z}_{j}^{-m}\right) \\
& =\prod_{i, j}\left(1+2 \zeta_{i} \bar{\zeta}_{j} D_{z_{i}}\left(-\frac{1}{z_{i} \bar{z}_{j}} \frac{1}{1+z_{i}^{-1} \bar{z}_{j}^{-1}}\right)\right) \\
& =\prod_{i, j}\left(1-2 \zeta_{i} \bar{\zeta}_{j} D_{z_{i}}\left(\frac{1}{1+z_{i} \bar{z}_{j}}\right)\right)=\prod_{i, j}\left(1-\zeta_{i} \bar{\zeta}_{j} \frac{1-z_{i} \bar{z}_{j}}{\left(1+z_{i} \bar{z}_{j}\right)^{2}}\right),
\end{aligned}
$$

where $D_{z}=\frac{1}{2}\left(z \partial_{z}+\partial_{z} z\right)$ is the Euler operator.

\subsection{Combinatorial meaning of $\mathrm{C}_{\lambda}(t)=\mathrm{C}_{\lambda}(1,0,0, \ldots)$}

Each partition with odd parts may be presented as $\lambda=\left(1^{m_{1}} 3^{m_{3}} 5^{m_{5}} \ldots\right)$ where $m_{i}$ is the multiplicity of the number $i$ (that means that the partition $\lambda$ contains the part equal to $i m_{i}$ times). The length $\ell(\lambda)$ of the partition $\lambda$ is equal to $\sum_{i=1,3,5, \ldots} m_{i}$, the weight is $|\lambda|=\sum_{i=1,3,5, \ldots} i m_{i}$.

Let us visualize this, in a similar way as in the papers $[5,18]$, as the one-dimensional semiinfinite lattice of cites (baskets) in our case numbered by odd positive integers. A basket number $i(i=1,3,5, \ldots)$ contains $m_{i}$ identical balls (and therefore the multiplicity $m_{i}$ may be also called the occupation number). Nonequivalent distributions of balls is in one-to-one correspondence with partitions from the set OP. (The length of a partition is equal to the number of balls, the ratio of the weight of the partition and the length of the partition may be considered as the location of the mass center of the balls).

Let us consider the following discrete time random process describing the creation of $\lambda \in \mathrm{OP}$ or, the same, of ball configurations. It starts with a given partition, say, $\mu$. (The case where $\mu=0$ describes the configuration where all baskets are empty at time $\mathrm{T}=0$ ). At each discrete time instant $\mathrm{T}=1,2,3, \ldots$ one of the following two possible events occurs with equal probability (A) either two balls are created in the leftmost basket (basket number 1), or (B) a ball chosen at random in any of baskets, say, in basket number $i$, is moved to the nearest basket to the right

(to the basket number $i+2$ ). It is clear that at each time step the weight of the related partition increases: $|\lambda| \rightarrow|\lambda|+2$, thus $|\lambda|=2 \mathrm{~T}$. A problem is to find a number of ways to create a given distribution $\lambda$ of the balls in baskets along the process described above in $\mathrm{T}=\frac{1}{2}|\lambda|$ steps. We denote this number $\mathrm{N}_{\mu \rightarrow \lambda}$.

Then we state that

$$
\mathrm{C}_{\lambda}(1,0,0,0, \ldots)=\left(\frac{1}{2}\right)^{\frac{1}{2} \ell(\lambda)} \frac{1}{\left(\frac{1}{2}|\lambda|\right) ! \frac{1}{d_{\lambda}}} \mathrm{N}_{0 \rightarrow \lambda}
$$

The proof follows from

$$
e^{J_{-1}}|0\rangle=|0\rangle+\sum_{\lambda \in \mathrm{OP}_{\mathrm{e}}}|\lambda\rangle \mathrm{C}_{\lambda}(-1,0,0,0, \ldots)=\sum_{\mathrm{T}=0,1,2, \ldots} \frac{1}{\mathrm{~T} !}\left(J_{-1}\right)^{\mathrm{T}}|0\rangle
$$


and from the detailed consideration of the action of

$$
J_{-1}=-\frac{1}{2} \phi_{\frac{1}{2}}^{2}+\phi_{-\frac{1}{2}} \phi_{\frac{3}{2}}-\phi_{-\frac{3}{2}} \phi_{\frac{5}{2}}+\cdots
$$

on basis Fock vectors (4.1) (see Appendix B), and from (4.8).

Now we write down the following formula for $C_{\lambda}(1,0,0, \ldots)$ obtained from (4.7):

$$
\mathrm{C}_{\lambda}(1,0,0, \ldots)=\frac{1}{d_{\lambda}} \prod_{i} \frac{1}{n_{i} ! n_{j} !} \operatorname{Hf}\left[\frac{1}{n_{i}+n_{j}+1}\right]
$$

where $n_{i}$ and $\lambda_{i}$ are related by (4.2), which yields

$$
\mathrm{N}_{0 \rightarrow \lambda}=2^{\frac{1}{2} \ell(\lambda)}\left(\frac{1}{2}|\lambda|\right) !\left(\prod_{i=1,3,5, \ldots} \frac{1}{m_{i} !}\right) \operatorname{Hf}\left[\frac{1}{n_{i} ! n_{j} !\left(n_{i}+n_{j}+1\right)}\right],
$$

where we recall that $m_{i}$ and $n_{i}$ we defined above: $\lambda=\left(1^{m_{1}} 3^{m_{3}} 5^{m_{5}} \ldots\right)$ and $\lambda=\left(\lambda_{1}, \ldots, \lambda_{2 k}\right)$ where $\ell(\lambda)=2 k$ is the length of the partition, $\lambda_{i}=2 n_{i}+1$.

For instance, take $\lambda=\left(1^{2 k}\right)$. Obviously there is only one way to create this configuration, $\mathrm{N}_{0 \rightarrow\left(1^{2 k}\right)}=1$. Indeed $\ell(\lambda)=|\lambda|=2 k, n_{i}=0, i=1, \ldots, 2 k$, and we obtain well-known identity

$$
1=\mathrm{N}_{0 \rightarrow\left(1^{2 k}\right)}=2^{k} k ! \frac{1}{(2 k) !}(2 k-1) ! !
$$

At last let us mention that $\mathrm{C}_{\lambda}(\mathbf{t})$ where $t_{i}=0$ except $i=\frac{1}{2}, 1$ may be related to the numbers $\mathrm{N}_{(1) \rightarrow \lambda}$, where $\lambda \in \mathrm{OP}_{\mathrm{o}}$.

\subsection{Polynomials $\mathrm{C}_{\lambda / \mu}$}

We define skew polynomials $\mathrm{C}_{\lambda / \mu}(\mathbf{t})$ as follows (cf. (2.27) and (4.10)):

$$
\mathrm{C}_{\lambda / \mu}(\mathbf{t}):=\langle\mu|\Gamma(\mathbf{t})| \lambda\rangle=\langle\lambda|\bar{\Gamma}(-\mathbf{t})| \mu\rangle .
$$

From this definition $C_{\lambda / \mu}$ vanishes unless $\mu \subseteq \lambda$. The same may be written as

$$
\Gamma(\mathbf{t})=\sum_{\lambda, \mu} \mathrm{C}_{\lambda / \mu}(\mathbf{t})|\mu\rangle\left\langle\lambda\left|, \quad \bar{\Gamma}(\mathbf{t})=\sum_{\lambda, \mu} \mathrm{C}_{\lambda / \mu}(-\mathbf{t})\right| \lambda\right\rangle\langle\mu| .
$$

If $\mathbf{t}=\mathbf{t}^{\prime}+\mathbf{t}^{\prime \prime}$ then $\Gamma(\mathbf{t})=\Gamma\left(\mathbf{t}^{\prime}\right) \Gamma\left(\mathbf{t}^{\prime \prime}\right)$ by inserting the unity operator $\sum_{\lambda \in P}|\lambda\rangle\langle\lambda|$ between $\Gamma\left(\mathbf{t}^{\prime}\right)$ and $\Gamma\left(\mathbf{t}^{\prime \prime}\right)$ we obtain

$$
\mathrm{C}_{\lambda}(\mathbf{t})=\sum_{\mu} \mathrm{C}_{\lambda / \mu}\left(\mathbf{t}^{\prime}\right) \mathrm{C}_{\mu}\left(\mathbf{t}^{\prime \prime}\right)
$$

This property is quite similar to the property of the Schur functions (see (5.9) in I of [11]).

One may relate $C_{\lambda / \mu}$ to the numbers $N_{\mu \rightarrow \lambda}$ described in the previous subsection.

\subsection{CKP tau function and polynomials $\mathrm{C}_{\lambda}$}

First of all we note that $C_{\lambda}(\mathbf{t})$ is not a solution of the Hirota bilinear equations, and, therefore is not a CKP tau function. However, due to (4.11) CKP tau functions (3.6) are series in $\mathrm{C}_{\lambda}(\mathbf{t})$ as follows

$$
\tau(\mathbf{t})=\sum_{\lambda \in \mathrm{OP}} g_{\lambda} \mathrm{C}_{\lambda}(\mathbf{t})
$$

where

$$
g_{\lambda}=\langle\lambda|g| 0\rangle .
$$


Example 1. Take $g=\exp \sum_{i>0} e^{-2 U_{i}} \phi_{i}^{2}$. Then

$$
\tau(\mathbf{t})=\sum_{\lambda} e^{-U_{\lambda}} \mathrm{C}_{\lambda}(\mathbf{t}) d_{\lambda} \prod_{i=1,3,5, \ldots} \frac{1}{k_{i} !},
$$

where the sum ranges over all $\lambda \in \mathrm{OP}$ whose parts have even multiplicities, $m_{i}=: 2 k_{i}$, i.e., of the form $\lambda=\left(1^{2 k_{1}} 3^{2 k_{3}} 5^{2 k_{5}} \ldots\right)$. The numbers $U_{\lambda}$ are defined as

$$
U_{\lambda}:=\sum_{i=1}^{k} U_{\frac{1}{2} \lambda_{i}} .
$$

The right-hand side of (4.16) may be compared to sums over partitions in [6] and in [14] dealing with tau functions of neutral and charged BKP hierarchies, respectively.

Example 2. Given a symmetric matrix $A$ and a partition $\lambda$ introduce numbers $A_{\lambda}$ according to the formula

$$
e^{\sum_{n, m>0} x_{n} A_{n m} x_{m}}=\sum_{\lambda \in P_{\mathrm{ev}}} A_{\lambda} x_{\lambda},
$$

where $\lambda=\left(\lambda_{1}, \ldots, \lambda_{k}\right)$ is a partition, and $x_{\lambda}=x_{\lambda_{1}} \cdots x_{\lambda_{k}}$, and where $P_{\mathrm{ev}}$ is a set of all partitions with even number of parts.

Then taking

$$
e^{\sum_{n \rightarrow m>0} \phi \frac{n}{2} A_{n m} \phi \frac{m}{2}}
$$

which is an exponential of a quadratic form of creation operators, we obtain

$$
\tau(\mathbf{t})=\langle 0|\Gamma(\mathbf{t}) g| 0\rangle=\sum_{\lambda \in P_{\mathrm{ev}}} A_{\lambda} \mathrm{C}_{\lambda}(\mathbf{t}) .
$$

Next we may write the CKP tau function as a double series over partitions

$$
\tau(\mathbf{t}, \bar{t})=\sum_{\lambda, \mu \in \mathrm{OP}} g_{\lambda, \mu} \mathrm{C}_{\lambda}(\mathbf{t}) \mathrm{C}_{\mu}(\bar{t})
$$

where

$$
g_{\lambda, \mu}=\langle\lambda|g| \mu\rangle .
$$

\section{Example 3.}

$$
\tau(\mathbf{t}, U, \bar{t})=\langle 0|\Gamma(\mathbf{t}) \mathbb{T}(U) \bar{\Gamma}(\bar{t})| 0\rangle=1+\sum_{\lambda \in \mathrm{OP}} e^{-U_{\lambda}} \mathrm{C}_{\lambda}(\mathbf{t}) \mathrm{C}_{\lambda}(-\bar{t}),
$$

where $U=\left(U_{\frac{1}{2}}, U_{\frac{3}{2}}, U_{\frac{5}{2}}, \ldots\right)$ is a set of constants, and

$$
\mathbb{T}(U):=\exp \sum_{0<i \in \frac{1}{2}+\mathbb{Z}}(-1)^{i+\frac{1}{2}} U_{i} \varphi_{i} \varphi_{-i},
$$

where the numbers $U_{\lambda}$ are defined by (4.17).

This example may be compared with the results of [15] and [13] devoted to TL and neutral BKP hypergeometric tau functions.

We can specify $\bar{t}$ in such a way that $\bar{t}_{i}=\frac{2}{i} z^{i}, i=1,3,5, \ldots$, and use (4.15). We thus obtain

$$
\tau(\mathbf{t}, U, \bar{t}(z))=1+\sum_{\lambda \in \mathrm{OP}_{\mathrm{ev}}} f_{\lambda} e^{-U_{\lambda}} \mathrm{C}_{\lambda}(\mathbf{t}), \quad f_{\lambda}:=(-1)^{\frac{1}{2}(|\lambda|+\ell(\lambda))} \frac{2}{d_{\lambda}} z^{\frac{1}{2}|\lambda|}(2 \ell(\lambda)-1) ! ! .
$$




\section{A Examples of CKP tau functions}

(I) One-soliton tau function is

$$
\left\langle 0\left|\Gamma(t) e^{\frac{a}{2} \phi(p) \phi(q)}\right| 0\right\rangle=\left(1-\frac{a}{p+q} e^{\sum\left(p^{n}+q^{n}\right) t_{n}}\right)^{-\frac{1}{2}} .
$$

(II) Another example is as follows

$$
\left\langle 0\left|\Gamma(t) e^{a \phi_{\frac{1}{2}}^{2}}\right| 0\right\rangle=\left(1-a t_{1}\right)^{-\frac{1}{2}},
$$

which may be also viewed as a solution of a heat equation

$$
\frac{\partial f\left(z_{\frac{1}{2}}, t_{1}\right)}{\partial t_{1}}=\frac{\partial^{2} f\left(z_{\frac{1}{2}}, t_{1}\right)}{\partial z_{\frac{1}{2}}^{2}}, \quad f\left(z_{\frac{1}{2}}, 0\right)=\exp a z_{\frac{1}{2}}^{2},
$$

which is

$$
f\left(z_{\frac{1}{2}}, t_{1}\right)=\exp t_{1} \partial_{\frac{1}{2}}^{2} \cdot \exp a z_{\frac{1}{2}}^{2}=\frac{1}{\sqrt{1-a t_{1}}} \exp \frac{a z_{\frac{1}{2}}^{2}}{1-a t_{1}}
$$

in the origin.

\section{B A realization of the algebra of free bosons (2.1)-(2.3)}

The simplest way to understand the action of $J_{-1}$ on a basis Fock vector may be as follows. Consider the realization of free boson algebra (2.1) via differentiation operators in auxiliary variables $z_{i}$ :

$$
\phi_{m+\frac{1}{2}}=z_{m+\frac{1}{2}}, \quad \phi_{-m-\frac{1}{2}}=(-)^{m} \partial_{m+\frac{1}{2}}, \quad m \geq 0,
$$

while the Fock space $F$ may be viewed as polynomial functions in the auxiliary variables $z_{i}$

$$
\left|\left(1^{m_{1}} 3^{m_{3}} 5^{m_{5}} \cdots\right)\right\rangle d_{\lambda}=z_{\frac{1}{2}}^{m_{1}} z_{\frac{3}{2}}^{m_{3}} z_{\frac{5}{2}}^{m_{5}} \cdots .
$$

The action of $J_{-1}$ on a basis Fock vector yields the following

$$
\begin{aligned}
& \left(-\frac{1}{2} z_{\frac{1}{2}}^{2}+z_{\frac{3}{2}} \partial_{\frac{1}{2}}+z_{\frac{5}{2}} \partial_{\frac{3}{2}}+\cdots\right) z_{\frac{1}{2}}^{m_{1}} z_{\frac{3}{2}}^{m_{3}} z_{\frac{5}{2}}^{m_{5}} \cdots \\
& \quad=-\frac{1}{2} z_{\frac{1}{2}}^{m_{1}+2} z_{\frac{3}{2}}^{m_{3}} z_{\frac{5}{2}}^{m_{5}} \cdots+m_{1} z_{\frac{1}{2}}^{m_{1}-1} z_{\frac{3}{2}}^{m_{3}+1} z_{\frac{5}{2}}^{m_{5}} \cdots+m_{3} z_{\frac{1}{2}}^{m_{1}} z_{\frac{3}{2}}^{m_{3}-1} z_{\frac{5}{2}}^{m_{5}+1} \cdots+\cdots
\end{aligned}
$$

As we see the action of $J_{-1}$ on a basis vector results in linear combination of basis vectors. The first term in the right-hand side of the last equality may be related to the event (A) (creation of a pair of balls in the basket 1 . This event is accomplished by the multiplication by the factor $-\frac{1}{2}$. Thus we get the general prefactor $(-1)^{\ell(\lambda)}$ for all configurations with $\ell(\lambda)$ number of the balls. Other terms in the right-hand side may be related to the event (B) where a chosen ball is moved to the right neighbor basket, each possibility has the weight 1 . The factors $m_{i}$ describe the fact that we can chose any of $m_{i}$ balls in the basket $i$ to move them to the right. 


\section{Bosonic KP tau function}

Here we shall briefly describe a bosonic KP tau function and its symplectic reduction to the bosonic CKP tau function.

Consider the following bosonic operators

$$
\left[p_{i}, q_{j}\right]=\delta_{i, j}, \quad i, j \in \frac{1}{2}+\mathbb{Z}
$$

The right and left Fock spaces will be defined via

$$
p_{i}|0\rangle=q_{-i}|0\rangle=0=\langle 0| q_{i}=\langle 0| p_{-i}, \quad i<0 .
$$

Let us define the expression $p_{i} q_{j}-\left\langle 0\left|p_{i} q_{j}\right| 0\right\rangle$ by $E_{i j}=: p_{i} q_{j}$ : These $E_{i j}$ may be considered as generators of $g l(\infty)$ algebra with a negative level.

Let us notice that for

$$
g:=\exp \sum_{i, j \in \frac{1}{2}+\mathbb{Z}} A_{i j}: p_{i} q_{j}:
$$

we have

$$
\sum_{i \in \frac{1}{2}+\mathbb{Z}} g p_{i} \otimes g q_{i}=\sum_{i \in \frac{1}{2}+\mathbb{Z}} p_{i} g \otimes q_{i} g
$$

Bosonic KP tau function may be defined as

$$
\tau^{K P}(\mathbf{t}):=\langle 0|\Gamma(\mathbf{t}) g| 0\rangle, \quad \Gamma(\mathbf{t}):=\exp \sum_{n=1}^{\infty} t_{n} \sum_{k \in \mathbb{Z}} p_{k} q_{k+n},
$$

where $\mathbf{t}=\left(t_{1}, t_{2}, \ldots\right)$ is a set of higher times. This tau function may be considered as a particular case of a supersymmetric KP tau function [9].

If we ask $g$ of (C.1) to be invariant under $S p(\infty)$ group then $g$ may be expressed in terms of the so-called symplectic bosons.

Symplectic bosons. Now between the bosons

$$
\phi_{i}=\frac{1}{\sqrt{2}}\left(p_{i}-(-1)^{i+\frac{1}{2}} q_{-i-1}\right), \quad \hat{\phi}_{i}=\frac{1}{\sqrt{2}}\left(p_{i}+(-1)^{i+\frac{1}{2}} q_{-i-1}\right)
$$

there are the following relations, cf. (2.1), (2.2):

$$
\left[\phi_{i}, \phi_{j}\right]=\left[\hat{\phi}_{j}, \hat{\phi}_{i}\right]=(-)^{j-\frac{1}{2}} \delta_{i,-j} \quad \text { and } \quad\left[\phi_{i}, \hat{\phi}_{j}\right]=0 \text {. }
$$

It is known (see [9]) that quadratic expressions $Z_{i j}=: \phi_{i} \phi_{j}$ : ordered via (2.6) may be considered as a realization for the generators of the $c_{\infty}$ algebra with a negative level (this fact may be verified with the help of (2.1) and (2.6)). The same is true for $\hat{Z}_{i j}=: \hat{\phi}_{i} \hat{\phi}_{j}$ :

It is straightforward to show that if $g$ of (C.1) is invariant under the $S p(\infty)$ group then $g=g_{1} \hat{g}_{1}$, where $g_{1}=\exp \left(\sum_{i, j \in \frac{1}{2}+\mathbb{Z}} A_{i j}: \phi_{i} \phi_{j}:\right)$ and $\hat{g}_{1}=\exp \left(\sum_{i, j \in \frac{1}{2}+\mathbb{Z}} A_{i j}: \hat{\phi}_{i} \hat{\phi}_{j}:\right) ;$ then, if all $t_{2 n-1}=0$, the KP bosonic tau function may be factorized: $\tau^{\mathrm{KP}}=\left(\tau^{\mathrm{CKP}}\right)^{-2}$, where $\tau^{\mathrm{CKP}}$ is of form (2.31), $\alpha=0$ (see also (3.6)). 


\section{Acknowledgements}

The authors thank J. Harnad for many discussions on related topics. This work has been partially supported by the European Union through the FP6 Marie Curie RTN ENIGMA (Contract no. MRTN-CT-2004-5652), the European Science Foundation Program MISGAM, by RFBR grant 11-01-00440-a, and by JSPS-RFBR grant 10-01-92104 JF.

\section{References}

[1] Aratyn H., van de Leur J.W., The CKP hierarchy and the WDVV prepotential, in Bilinear Integrable Systems: from Classical to Quantum, Continuous to Discrete, NATO Sci. Ser. II Math. Phys. Chem., Vol. 201, Springer, Dordrecht, 2006, 1-11, nlin.SI/0302004.

[2] Date E., Jimbo M., Kashiwara M., Miwa T., Transformation groups for soliton equations. III. Operator approach to the Kadomtsev-Petviashvili equation, J. Phys. Soc. Japan 50 (1981), 3806-3812.

[3] Date E., Jimbo M., Kashiwara M., Miwa T., Transformation groups for soliton equations. VI. KP hierarchies of orthogonal and symplectic type, J. Phys. Soc. Japan 50 (1981), 3813-3818.

[4] Date E., Kashiwara M., Jimbo M., Miwa T., Transformation groups for soliton equations, in Nonlinear Integrable Systems - Classical Theory and Quantum Theory (Kyoto, 1981), World Sci. Publishing, Singapore, 1983, 39-119.

[5] Harnad J., Orlov A.Yu., Fermionic construction of tau functions and random processes, Phys. D 235 (2007), 168-206, arXiv:0704.1157.

[6] Harnad J., van de Leur J.W., Orlov A.Yu., Multiple sums and integrals as neutral BKP tau functions, Theoret. and Math. Phys. 168 (2011), 951-962, arXiv:1101.4216.

[7] Ishikawa M., Kawamuko H., Okada S., A Pfaffian-Hafnian analogue of Borchardt's identity, math.CO/0408364.

[8] Kac V., Vertex algebras for beginners, University Lecture Series, Vol. 10, 2nd ed., American Mathematical Society, Providence, RI, 1998.

[9] Kac V.G., van de Leur J.W., Super boson-fermion correspondence of type B, in Infinite-Dimensional Lie Algebras and Groups (Luminy-Marseille, 1988), Adv. Ser. Math. Phys., Vol. 7, World Sci. Publ., Teaneck, NJ, 1989, 369-406.

[10] Kac V.G., van de Leur J.W., The $n$-component KP hierarchy and representation theory, J. Math. Phys. 44 (2003), 3245-3293, hep-th/9308137.

[11] Macdonald I.G., Symmetric functions and Hall polynomials, 2nd ed., Oxford Mathematical Monographs, The Clarendon Press Oxford University Press, New York, 1995.

[12] Nimmo J.J.C., Hall-Littlewood symmetric functions and the BKP equation, J. Phys. A: Math. Gen. 23 (1990), 751-760.

[13] Orlov A.Yu., Hypergeometric functions related to Schur Q-polynomials and the BKP equation, Theoret. and Math. Phys. 137 (2003), 1574-1589, math-ph/0302011.

[14] Orlov A.Yu., Shiota T., Takasaki K., Pfaffian structures and certain solutions to BKP hierarchies. I. Sums over partitions, arXiv:1201.4518.

[15] Orlov A.Yu., Scherbin D.M., Multivariate hypergeometric functions as $\tau$-functions of Toda lattice and Kadomtsev-Petviashvili equation, Phys. D 152/153 (2001), 51-65, math-ph/0003011.

[16] Sato M., Soliton equations as dynamical systems on an infinite dimensional Grassmann manifolds, in Random Systems and Dynamical Systems (Kyoto, 1981), RIMS Kokyuroku, Vol. 439, Kyoto, 1981, 30-46.

[17] Takasaki K., Initial value problem for the Toda lattice hierarchy, in Group Representations and Systems of Differential Equations (Tokyo, 1982), Adv. Stud. Pure Math., Vol. 4, North-Holland, Amsterdam, 1984, $139-163$.

[18] van de Leur J.W., Orlov A.Yu., Random turn walk on a half line with creation of particles at the origin, Phys. Lett. A 373 (2009), 2675-2681, arXiv:0801.0066.

[19] You Y., Polynomial solutions of the BKP hierarchy and projective representations of symmetric groups, in Infinite-Dimensional Lie Algebras and Groups (Luminy-Marseille, 1988), Adv. Ser. Math. Phys., Vol. 7, World Sci. Publ., Teaneck, NJ, 1989, 449-464. 